MATHEMATICS OF COMPUTATION

Volume 72, Number 241, Pages 83-104

S 0025-5718(01)01413-2

Article electronically published on December 5, 2001

\title{
QUADRATIC FINITE ELEMENT APPROXIMATION OF THE SIGNORINI PROBLEM
}

\author{
Z. BELHACHMI AND F. BEN BELGACEM
}

\begin{abstract}
Applying high order finite elements to unilateral contact variational inequalities may provide more accurate computed solutions, compared with linear finite elements. Up to now, there was no significant progress in the mathematical study of their performances. The main question is involved with the modeling of the nonpenetration Signorini condition on the discrete solution along the contact region. In this work we describe two nonconforming quadratic finite element approximations of the Poisson-Signorini problem, responding to the crucial practical concern of easy implementation, and we present the numerical analysis of their efficiency. By means of Falk's Lemma we prove optimal and quasi-optimal convergence rates according to the regularity of the exact solution.
\end{abstract}

\section{InTRODUCTION AND FUNCTIONAL TOOLS}

Contact problems are in the heart of a high number of mechanical structures and also have a great importance in hydrostatics and thermostatics. Among them, unilateral contact, typically represented by Signorini's model, causes some specific difficulties, on both theoretical and approximation grounds. We refer to [11], [13] and [19] for mathematical foundation. Much attention has been devoted to the numerical simulation of variational inequalities modeling unilateral contact, by finite elements, either from the accuracy point of view (see 16, [18, 24 and references therein) or for developing efficient algorithms to solve the final minimization problem (see [13, 2]). The hardest task is the discrete modeling of the Signorini unilateral condition, which, most often, is not fulfilled exactly by the computed solution (the normal displacement) — even though for linear finite elements the conforming method is also used by practitioners, because it is easy to implement, and turns out to be reliable. Then, the construction of the finite dimensional closed convex cone, on which the approximated inequality is set, results in a nonconforming approach. Nevertheless, the numerical analysis realized on the linear finite element methods and under reasonable regularity assumptions on the exact solution yields satisfactory convergence rates when compared to those expected for the general finite element theory. We refer in particular to [3] and to [4] for quasi-optimal studies.

When high accuracy is needed, a possible way to respond to such a request consists in refining the mesh used with linear finite elements. An alternative is to resort

Received by the editor April 20, 2000 and, in revised form, April 10, 2001.

2000 Mathematics Subject Classification. Primary 35J85, 73J05.

Key words and phrases. Variational inequalities, Signorini problem, quadratic finite element, error estimates.

(C)2001 American Mathematical Society 
to quadratic finite elements, which proved to perform for linear partial differential equations governing the temperature diffusion within a conducting body or the elastic displacement of a deformable structure. The difference between conforming and nonconforming methods becomes, here, very important for practical facts. Indeed, the exact unilateral condition is not at all easy to take into account in a computing code, and it is better to enforce such a condition on the computed solution at only a finite number of degrees of freedom. The purpose of this contribution is to describe two efficient ways to satisfy (in a weak sense) the unilateral condition, ways that are easily handled in a practical context. The numerical analysis detailed here provides the desired asymptotic convergence rates.

An outline of the paper is as follows. In Section 2 we write a variational formulation of the Poisson-Signorini problem. Section 3 is a description of the first quadratic finite element approximation of the resulting inequality; the contact condition is enforced on the discrete solution values at the vertices of the elements that are located in the contact region and on its momenta in each of these elements. The numerical analysis of this discretization is detailed in Section 4, where optimal convergence rates are exhibited when reasonable regularity is assumed on the exact solution. Section 5 is dedicated to the study of the more natural numerical contact model, where nonpenetration is imposed at all the Lagrange nodes of the contact zone. This second method performs as well as the first one. The main difference between them is that the first method is suitable when we are interested in checking the contact condition on the normal constraint, while the second is more appropriate when we prefer to check the Signorini condition on the normal displacement.

Notation. Let $\Omega \subset \mathbb{R}^{2}$ be a Lipschitz domain with generic point $\boldsymbol{x}$. The Lebesgue space $L^{p}(\Omega)$ is endowed with the norm: $\forall \psi \in L^{p}(\Omega)$,

$$
\|\psi\|_{L^{p}(\Omega)}=\left(\int_{\Omega}|\psi(\boldsymbol{x})|^{p} d \boldsymbol{x}\right)^{\frac{1}{p}} .
$$

We make a constant use of the standard Sobolev space $H^{m}(\Omega), m \geq 1$, provided with the norm

$$
\|\psi\|_{H^{m}(\Omega)}=\left(\sum_{0 \leq|\alpha| \leq m}\left\|\partial^{\alpha} \psi\right\|_{L^{2}(\Omega)}^{2}\right)^{\frac{1}{2}}
$$

where $\alpha=\left(\alpha_{1}, \alpha_{2}\right)$ is a multi-index in $\mathbb{N}^{2}$ and the symbol $\partial^{\alpha}$ represents a partial derivative $\left(H^{0}(\Omega)=L^{2}(\Omega)\right)$. The fractional order Sobolev space $H^{\nu}(\Omega), \nu \in \mathbb{R}_{+} \backslash \mathbb{N}$, is defined by the norm

$$
\|\psi\|_{H^{\nu}(\Omega)}=\left(\|\psi\|_{H^{m}(\Omega)}^{2}+\sum_{|\alpha|=m} \int_{\Omega} \int_{\Omega} \frac{\left(\partial^{\alpha} \psi(\boldsymbol{x})-\partial^{\alpha} \psi(\boldsymbol{y})\right)^{2}}{|\boldsymbol{x}-\boldsymbol{y}|^{2+2 \theta}}\right)^{\frac{1}{2}},
$$

where $\nu=m+\theta, m$ is the integer part of $\nu$ and $\theta \in] 0,1[$ is the decimal part (see [1] [14], [21]). The closure in $H^{\nu}(\Omega)$ of $\mathcal{D}(\Omega)$ is denoted $H_{0}^{\nu}(\Omega)$, where $\mathcal{D}(\Omega)$ is the space of infinitely differentiable functions whose support is contained in $\Omega$.

For any portion $\gamma$ of the boundary $\partial \Omega$ and any $\nu>0$, the Hilbert space $H^{\nu}(\gamma)$ is defined as the range of $H^{\nu+\frac{1}{2}}(\Omega)$ by the trace operator; it is then endowed with the image norm

$$
\|\psi\|_{H^{\nu}(\gamma)}=\inf _{\chi \in H^{\nu+\frac{1}{2}}(\Omega), \chi \mid \gamma=\psi}\|\chi\|_{H^{\nu+\frac{1}{2}}(\Omega)}
$$


When $\gamma$ is sufficiently regular, one can directly write down an explicit norm of $H^{\nu}(\gamma)$, while for polygonal lines - which will be the situation of interest in this work - it turns out to be more complicated to have an explicit norm, especially for $\nu \geq \frac{3}{2}$. Nevertheless, we can use the results given in Theorem 1.4.6 in [14] to obtain an explicit norm; some compatibility conditions should be taken into account at the vicinity of the corner point of $\gamma$. The space $H^{\nu}(\gamma)^{\prime}$ stands for the topological dual space of $H^{\nu}(\gamma)$ and the duality pairing is denoted $\langle., .\rangle_{\nu, \gamma}$. Moreover, if an interval $\gamma$ is the disjoint union of subintervals $\gamma_{k}\left(1 \leq k \leq k^{*}\right)$, then, $\forall \psi \in H^{\nu}(\gamma)$,

$$
\sum_{k=1}^{k^{*}}\|\psi\|_{H^{\nu}\left(\gamma_{k}\right)}^{2} \leq\|\psi\|_{H^{\nu}(\gamma)}^{2} .
$$

The inequality is still valid when the norm $H^{\nu}(\gamma)$ is replaced by the semi-norm. To be complete with the Sobolev functional tools used hereafter, recall that for $\nu>\frac{3}{2}$, the trace operator

$$
T: \psi \mapsto\left(\psi_{\mid \partial \Omega},\left(\frac{\partial \psi}{\partial \boldsymbol{n}}\right)_{\mid \partial \Omega}\right)
$$

is continuous from $H^{\nu}(\Omega)$ onto $H^{\nu-\frac{1}{2}}(\partial \Omega) \times H^{\nu-\frac{3}{2}}(\partial \Omega)$ (see [14]). Otherwise, if $1 \leq \nu \leq \frac{3}{2}$, define the space $X^{\nu}(\Omega)$ to be

$$
X^{\nu}(\Omega)=\left\{\psi \in H^{\nu}(\Omega), \Delta \psi \in L^{2}(\Omega)\right\},
$$

equipped with the graph norm

$$
\|\psi\|_{X^{\nu}(\Omega)}=\left(\|\psi\|_{H^{\nu}(\Omega)}^{2}+\|\Delta \psi\|_{L^{2}(\Omega)}^{2}\right)^{\frac{1}{2}} .
$$

Then the trace operator $T$ is continuous from $X^{\nu}(\Omega)$ onto $H^{\nu-\frac{1}{2}}(\partial \Omega) \times H^{\frac{3}{2}-\nu}(\partial \Omega)$. Sometimes, we need to use the Hölder space $\mathcal{C}^{0, \alpha}(\gamma), 0<\alpha \leq 1$, defined as

$$
\mathcal{C}^{0, \alpha}(\gamma)=\left\{\psi \in \mathcal{C}^{0}(\gamma), \quad\|\psi\|_{\mathcal{C}^{0, \alpha}(\gamma)}=\sup _{\boldsymbol{x} \in \gamma}|\psi(\boldsymbol{x})|+\sup _{\boldsymbol{x}, \boldsymbol{y} \in \gamma} \frac{|\psi(\boldsymbol{x})-\psi(\boldsymbol{y})|}{|\boldsymbol{x}-\boldsymbol{y}|^{\alpha}}<\infty\right\} .
$$

\section{The Continuous Poisson-Signorini Problem}

Let $\Omega$ be a Lipschitz bounded domain in $\mathbb{R}^{2}$. The boundary $\partial \Omega$ is a union of three nonoverlapping portions $\Gamma_{u}, \Gamma_{g}$ and $\Gamma_{C}$. The vertices of $\Gamma_{C}$ are $\left\{\boldsymbol{c}_{1}, \boldsymbol{c}_{2}\right\}$ and those of $\Gamma_{u}$ are $\left\{\boldsymbol{c}_{1}^{\prime}, \boldsymbol{c}_{2}^{\prime}\right\}$. The part $\Gamma_{u}$ of nonzero (surface) measure is subjected to Dirichlet conditions, while on $\Gamma_{g}$ a Neumann condition is prescribed, and $\Gamma_{C}$ is the candidate to be in contact with a rigid obstacle. To avoid technicalities arising from the special Sobolev space $H_{00}^{\frac{1}{2}}\left(\Gamma_{C}\right)$, we assume that $\Gamma_{u}$ and $\Gamma_{C}$ do not touch.

For a given data $f \in L^{2}(\Omega)$ and $g \in H^{\frac{1}{2}}\left(\Gamma_{g}\right)^{\prime}$, the Signorini problem consists in finding $u$ that verifies, in a distributional sense,

$$
\begin{aligned}
& -\Delta u=f \quad \text { in } \Omega, \\
& u=0 \quad \text { on } \Gamma_{u}, \\
& \frac{\partial u}{\partial \boldsymbol{n}}=g \quad \text { on } \Gamma \text {, } \\
& u \geq 0, \quad \frac{\partial u}{\partial \boldsymbol{n}} \geq 0, \quad u \frac{\partial u}{\partial \boldsymbol{n}}=0 \quad \text { on } \Gamma_{C},
\end{aligned}
$$

where $\boldsymbol{n}$ is the outward unit normal to $\partial \Omega$. Most often, the modeling of the contact condition is formulated using a gap function $\alpha$ defined on $\Gamma_{C}$, so that instead of 
$u \geq 0$ and the saturation condition $u \frac{\partial u}{\partial \boldsymbol{n}}=0$ we have $u-\alpha \geq 0$ and $(u-\alpha) \frac{\partial u}{\partial \boldsymbol{n}}=$ 0 on the contact zone $\Gamma_{C}$ (see [11]). As the whole subsequent analysis can be extended straightforwardly to the case where $\alpha$ does not vanish, we choose, only for conciseness, to take $\alpha=0$.

The functional framework well suited to solve problem (2.1)-(2.4) consists in working with the subspace $H_{0}^{1}\left(\Omega, \Gamma_{u}\right)$ of $H^{1}(\Omega)$ made up of functions that vanish at $\Gamma_{u}$. The semi-norm is actually, by the Poincaré inequality, a norm in $H_{0}^{1}\left(\Omega, \Gamma_{u}\right)$ equivalent to the norm of $H^{1}(\Omega)$. In the weak formulation, the unilateral contact condition on $\Gamma_{C}$ is taken into account by incorporating it in the closed convex cone

$$
K(\Omega)=\left\{v \in H_{0}^{1}\left(\Omega, \Gamma_{u}\right), \quad v_{\mid \Gamma_{C}} \geq 0, \text { a.e. }\right\} .
$$

The primal variational principle for the Signorini problem produces the variational inequality: find $u \in K(\Omega)$ such that

$$
a(u, v-u) \geq L(v-u), \quad \forall v \in K(\Omega) .
$$

In (2.5) we have set

$$
\begin{aligned}
a(u, v) & =\int_{\Omega} \nabla u \nabla v d \boldsymbol{x}, \\
L(v) & =\int_{\Omega} f v d \boldsymbol{x}+\langle g, v\rangle_{\frac{1}{2}, \Gamma_{g}} .
\end{aligned}
$$

By Stampacchia's Theorem (see [16]), the weak problem (2.5) is well posed and has only one solution in $K(\Omega)$ that depends continuously on the data $(f, g)$.

Remark 2.1. In the variational formulation, the mathematical sense given to conditions (2.3) and (2.4) is as follows:

$$
\begin{aligned}
& \left\langle\frac{\partial u}{\partial \boldsymbol{n}}, v\right\rangle_{\frac{1}{2}, \partial \Omega}-\langle g, v\rangle_{\frac{1}{2}, \Gamma_{g}} \geq 0, \quad \forall v \in H_{00}^{\frac{1}{2}}\left(\partial \Omega, \Gamma_{u}\right), \quad v_{\mid \Gamma_{C}} \geq 0, \\
& \left\langle\frac{\partial u}{\partial \boldsymbol{n}}, u\right\rangle_{\frac{1}{2}, \partial \Omega}-\langle g, u\rangle_{\frac{1}{2}, \Gamma_{g}}=0,
\end{aligned}
$$

where $H_{00}^{\frac{1}{2}}\left(\partial \Omega, \Gamma_{u}\right)$ is the subspace of $H^{\frac{1}{2}}(\partial \Omega)$ of the functions that vanish on $\Gamma_{u}$. Roughly, (2.6) says that $\frac{\partial u}{\partial \boldsymbol{n}}=g$ on $\Gamma_{g}$ and $\frac{\partial u}{\partial \boldsymbol{n}} \geq 0$ on $\Gamma_{C}$, while (2.7) expresses the saturation condition $u \frac{\partial u}{\partial \boldsymbol{n}}=0$ on $\Gamma_{C}$.

Remark 2.2. Apart from the strong singularities created by changing from the Dirichlet to the Neumann condition around the vertices $\left\{\boldsymbol{c}_{1}^{\prime}, \boldsymbol{c}_{2}^{\prime}\right\}$, it is now well known that the unilateral condition may generate some singular behavior in the vicinity of $\Gamma_{C}$ even for very regular data $(f, g)$ and a very smooth boundary $\partial \Omega$. For example, if $f \in H^{1}(\Omega)$, the solution $u$ may not be of class $H^{3}$ around $\left(\Gamma_{C} \backslash\left\{\boldsymbol{c}_{1}, \boldsymbol{c}_{2}\right\}\right)$ (see [22]). The reason is the following. Let $\boldsymbol{m}$ be a point of $\Gamma_{C}$ where the constraints change from binding to nonbinding. Then the singularity $S_{\boldsymbol{m}}(r, \theta)=r^{\frac{3}{2}} \sin \left(\frac{3}{2} \theta\right) \varphi(r)$ $((r, \theta)$ are the polar coordinates with origin $\boldsymbol{m}$ and $\varphi$ is a smooth function with compact support and equal to 1 in the vicinity of $\boldsymbol{m}$ ) is involved in the decomposition of the solution on the Dirichlet-Neumann singular functions. The first singularity $r^{\frac{1}{2}} \sin \left(\frac{1}{2} \theta\right) \psi(r)$ is cancelled because it fails to satisfy the Signorini condition (the nonnegativity of both $S_{\boldsymbol{m}}$ and $\frac{\partial S_{m}}{\partial \boldsymbol{n}}$ ). The best we can expect is to obtain $u \in H^{\sigma}\left(V_{\Gamma_{C}}\right)$ with $\sigma<\frac{5}{2}$ and $V_{\Gamma_{C}}$ an open set containing $\Gamma_{C}$ (see [22]). 


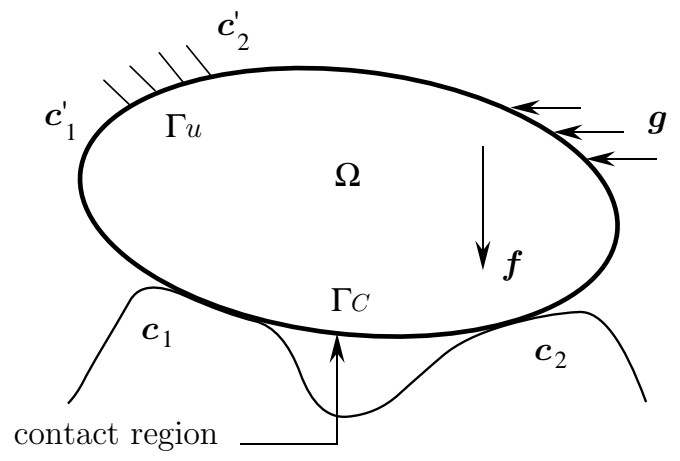

FIGURE 2.1.

Remark 2.3. The Signorini problem has many important applications, particularly in mechanics. In deformable structure mechanics, the displacement of a body $\Omega$ (represented in Figure 2.1) supported by a frictionless rigid foundation $\Gamma_{C}$, fixed along a part $\Gamma_{\boldsymbol{u}}$ of the border and subjected to external forces $\boldsymbol{f}_{\mid \Omega}$ and $\boldsymbol{g}_{\mid \Gamma_{\boldsymbol{g}}}$, is a solution of the following problem:

$$
\begin{aligned}
-\boldsymbol{d i v} \sigma(\boldsymbol{u}) & =\boldsymbol{f} & & \text { in } \Omega, \\
\sigma(\boldsymbol{u}) \boldsymbol{n} & =\boldsymbol{g} & & \text { on } \Gamma_{\boldsymbol{g}}, \\
\boldsymbol{u} & =0 & & \text { on } \Gamma_{\boldsymbol{u}} .
\end{aligned}
$$

The bold symbol $\boldsymbol{d i v}$ denotes the divergence operator of a tensor function and is defined as $\boldsymbol{d i v} \sigma=\left(\frac{\partial \sigma_{i j}}{\partial x_{j}}\right)_{i}$. The stress tensor is obtained from the displacement through the constitutive law $\sigma(\boldsymbol{u})=A(\boldsymbol{x}) \varepsilon(\boldsymbol{u})$, where $A(\boldsymbol{x}) \in\left(L^{\infty}(\Omega)\right)^{16}$, the Hook tensor, is of fourth order, symmetric and elliptic. Finally, to close the system, frictionless contact conditions are needed on $\Gamma_{C}$. Denoting by $\sigma_{\boldsymbol{n}}$ the normal component of $(\sigma \boldsymbol{n})$ and by $\sigma_{\boldsymbol{t}}$ its tangential component, the contact conditions are formulated as follows:

$$
\begin{aligned}
\boldsymbol{u} . \boldsymbol{n} \leq 0, \quad \sigma_{\boldsymbol{n}} & \leq 0, \quad \sigma_{\boldsymbol{n}}(\boldsymbol{u} . \boldsymbol{n})=0, \\
\sigma_{\boldsymbol{t}} & =0 .
\end{aligned}
$$

The weak problem is set on the closed convex set

$$
\boldsymbol{K}(\Omega)=\left\{\boldsymbol{v} \in H_{0}^{1}\left(\Omega, \Gamma_{\boldsymbol{u}}\right)^{2}, \boldsymbol{v} \cdot \boldsymbol{n}_{\mid \Gamma_{C}} \leq 0, \text { a.e. }\right\} .
$$

It reads as follows: find $\boldsymbol{u} \in \boldsymbol{K}(\Omega)$ such that

$$
\int_{\Omega} A \varepsilon(\boldsymbol{u}): \varepsilon(\boldsymbol{v}-\boldsymbol{u}) d \boldsymbol{x} \geq \int_{\Omega} \boldsymbol{f} .(\boldsymbol{v}-\boldsymbol{u}) d \boldsymbol{x}+\int_{\Gamma_{g}} \boldsymbol{g} \cdot(\boldsymbol{v}-\boldsymbol{u}) d \Gamma, \quad \forall \boldsymbol{v} \in \boldsymbol{K}(\Omega) .
$$

In the linear elasticity context, where the body undergoes small displacements with the strain tensor $\varepsilon(\boldsymbol{u})=\frac{1}{2}\left(\nabla \boldsymbol{u}+(\nabla \boldsymbol{u})^{T}\right)$, this variational problem has the same properties as the Signorini problem (2.5) that we intend to study in detail. Then, our whole numerical analysis is extended as well to the unilateral contact elasticity problem. 


\section{QuAdRATiC FINITE ELEMENT DisCRETIZATION: FIRST NUMERICAL MODEL}

The convergence rate of the finite element approximation of the Signorini problem depends on the regularity of the solution $u$. In practice, it may occur that $u$ belongs to a more regular space than $H^{2}$, at least around $\Gamma_{C}$ (see Remark 2.2 ). Therefore, the numerical simulation of problem (2.5) based on affine finite elements fails to profit from the full regularity of $u_{\mid V_{\Gamma_{C}}}$ (see [16], [3], 4]). Indeed, in this case the effective useful regularity is that of $H^{2}$. To alleviate this limitation we resort to quadratic finite elements for the discretization of the weak Signorini problem.

For the description of the method, for simplicity and to avoid more technicalities the shape of the domain $\Omega$ is assumed polygonal, so that it can be exactly covered by rectilinear finite elements. The generalization to curved domains is done following [15] and is not addressed here. For any given discretization parameter $h>0$, let there be given a partition $\mathcal{T}_{h}$ of $\Omega$ into triangles with a maximum size $h$,

$$
\bar{\Omega}=\bigcup_{\kappa \in \mathcal{T}_{h}} \bar{\kappa} .
$$

The motivation of the choice of triangular finite elements is that they are more widely used than quadrangular ones. However, the whole analysis set forth here applies as well to the quadrangular finite elements.

The family $\left(\mathcal{T}_{h}\right)_{h}$ is assumed to be $\mathcal{C}^{0}$-regular in the classical sense [9]. Moreover $\mathcal{T}_{h}$ is built in such a way that $\left\{\boldsymbol{c}_{1}, \boldsymbol{c}_{2}, \boldsymbol{c}_{1}^{\prime}, \boldsymbol{c}_{2}^{\prime}\right\}$ coincide with the vertices of some elements. For any $\kappa \in \mathcal{T}_{h}, \mathcal{P}_{2}(\kappa)$ stands for the set of polynomials of total degree $\leq 2$. Then we introduce the finite dimensional subspace $X_{h}(\Omega)$ of $H_{0}^{1}\left(\Omega, \Gamma_{u}\right)$ :

$$
X_{h}(\Omega)=\left\{v_{h} \in \mathcal{C}(\bar{\Omega}), \forall \kappa \in \mathcal{T}_{h}, v_{h \mid \kappa} \in \mathcal{P}_{2}(\kappa), v_{h \mid \Gamma_{u}}=0\right\}
$$

Let $\Sigma_{h}$ denote the set of all corners and midpoints of edges of the elements $\kappa$ in $\mathcal{T}_{h}$. Set $\Xi_{h}=\Sigma_{h} \backslash \Gamma_{u}$; then $\left(\Omega, X_{h}(\Omega), \Xi_{h}\right)$ is unisolvent. Furthermore, if $\left(\mathcal{I}_{h}\right)$ stands for the standard Lagrange interpolation operator, then for any $\mu(0 \leq \mu \leq 1)$ and any $\nu(1<\nu \leq 3)$ we have, for all $v \in H^{\nu}(\Omega)$,

$$
\left\|v-\mathcal{I}_{h} v\right\|_{H^{\mu}(\Omega)} \leq C h^{\nu-\mu}\|v\|_{H^{\nu}(\Omega)} .
$$

Realizing a conforming approximation requires one to impose the nonpenetration condition $u_{h} \geq 0$ everywhere in $\Gamma_{C}$. An essential drawback of this model arises in the implementation. We do not see how to take into account, in an easy way, the condition $u_{h \mid \Gamma_{C}} \geq 0$ in a computing code. To overcome this complication, it is better to enforce nonnegativity on a finite number of degrees of freedom "located" on $\Gamma_{C}$, which, most often, results in a nonconforming finite element approach. The construction of the discrete convex cone requires the introduction of some more notation connected with the contact zone. Due to the $\mathcal{C}^{0}$-regularity hypothesis, the boundary inherits a regular mesh $\mathcal{T}_{h}^{\partial \Omega}$, the elements of which are complete edges of the triangles $\kappa \in \mathcal{T}_{h}$. The trace of $\mathcal{T}_{h}^{\partial \Omega}$ on $\Gamma_{C}$ results in a mesh denoted by $\mathcal{T}_{h}^{C}$ and is characterized by the subdivision $\left(\boldsymbol{x}_{i}^{C}\right)_{0 \leq i \leq i^{*}}$ with $\boldsymbol{x}_{0}^{C}=\boldsymbol{c}_{1}$ and $\boldsymbol{x}_{i^{*}}^{C}=\boldsymbol{c}_{2}$, $\left(t_{i}=\right] \boldsymbol{x}_{i}^{C}, \boldsymbol{x}_{i+1}^{C}[)_{0 \leq i \leq i^{*}-1}$ for its elements, and the middle node of $t_{i}$ is denoted by $\boldsymbol{x}_{i+\frac{1}{2}}^{C}$.

Our first choice consists to enforce nonnegativity on the values of $u_{h}$ at the vertices $\left(\boldsymbol{x}_{i}^{C}\right)_{0 \leq i \leq i^{*}}$ and on its momentum on the elements $\left(t_{i}\right)_{0 \leq i \leq i^{*}-1}$. Then, we 
work with the finite dimensional closed convex cone,

$$
\begin{aligned}
& K_{h}(\Omega)=\left\{v_{h} \in X_{h}(\Omega), \quad v_{h}\left(\boldsymbol{x}_{i}^{C}\right) \geq 0, \quad \forall i\left(0 \leq i \leq i^{*}\right),\right. \\
& \left.\int_{t_{i}} v_{h} d \Gamma \geq 0, \quad \forall i\left(0 \leq i \leq i^{*}-1\right)\right\} .
\end{aligned}
$$

For our purpose we need to introduce an operator $\left(\mathcal{J}_{h}\right)$ more appropriate than $\left(\mathcal{I}_{h}\right)$; it is defined by the following degrees of freedom:

$$
(v(\boldsymbol{x}))_{\boldsymbol{x} \in \Xi_{h} \backslash \Gamma_{C}}, \quad\left(v\left(\boldsymbol{x}_{i}^{C}\right)\right)_{0 \leq i \leq i^{*}}, \quad\left(\int_{t_{i}} v(\boldsymbol{x}) d \Gamma\right)_{0 \leq i \leq i^{*}-1} .
$$

The operator $\left(\mathcal{J}_{h}\right)$ has similar localization properties as $\left(\mathcal{I}_{h}\right)$, i.e., $\left(\mathcal{J}_{h} v\right)_{\mid \kappa}$ depends only on $v_{\mid \kappa}, \forall \kappa \in \mathcal{T}_{h}$. In addition, using the Bramble-Hilbert Theorem, the following error estimate holds, for any $\mu(0 \leq \mu \leq 1)$ and for any $\nu(1<\nu \leq 3)$ there exists a constant $C>0$ such that, $\forall v \in H^{\nu}(\Omega)$,

$$
\left\|v-\mathcal{J}_{h} v\right\|_{H^{\mu}(\Omega)} \leq C h^{\nu-\mu}\|v\|_{H^{\nu}(\Omega)} .
$$

It is easy to see that for any $v \in K(\Omega) \cap \mathcal{C}^{0}(\bar{\Omega})$ we have $\left(\mathcal{J}_{h} v\right) \in K_{h}(\Omega)$. Let us also remark that there is no reason why $\left(\mathcal{J}_{h} v\right)_{\mid \Gamma_{C}} \geq 0$. Another essential feature of the operator $\mathcal{J}_{h}$ is the optimality of the approximation estimates it provides on $\Gamma_{C}$, in the dual Sobolev spaces. Before discussing them, we need to introduce some additional functional tools. Denote

$$
M_{h}\left(\Gamma_{C}\right)=\left\{\psi_{h} \in L^{2}\left(\Gamma_{C}\right), \quad \psi_{h \mid t_{i}} \in \mathcal{P}_{0}\left(t_{i}\right), \quad \forall i\left(0 \leq i \leq i^{*}-1\right)\right\},
$$

and let $\pi_{h}^{C}$ be the projection defined on $H^{\mu}\left(\Gamma_{C}\right)^{\prime} \rightarrow M_{h}\left(\Gamma_{C}\right)$ for any $\mu\left(0 \leq \mu<\frac{1}{2}\right)$ by, $\forall \psi \in H^{\mu}\left(\Gamma_{C}\right)^{\prime}$,

$$
\left\langle\psi-\pi_{h}^{C} \psi, \chi_{h}\right\rangle_{\mu, \Gamma_{C}}=0, \quad \forall \chi_{h} \in M_{h}\left(\Gamma_{C}\right) .
$$

Notice that if $\psi \in L^{2}\left(\Gamma_{C}\right)$ then $\left(\pi_{h}^{C} \psi\right)_{\mid t_{i}}=\frac{1}{\left|t_{i}\right|} \int_{t_{i}} \psi d \Gamma$. As we know, for any $\nu, \mu(0 \leq \mu, \nu \leq 1)$ we have, $\forall \psi \in H^{\nu}\left(\Gamma_{C}\right)$,

$$
\left\|\psi-\pi_{h}^{C} \psi\right\|_{H^{\mu}\left(\Gamma_{C}\right)^{\prime}} \leq C h^{\nu+\mu}\|\psi\|_{H^{\nu}\left(\Gamma_{C}\right)} .
$$

Besides, $\pi_{h}^{C}$ satisfies some nonstandard approximation result (see Lemma 7.2). Indeed, for $\mu\left(0 \leq \mu<\frac{1}{2}\right)$ and $\nu\left(\frac{1}{2}<\nu \leq 1\right)$ we have, $\forall \psi \in H^{\mu}\left(\Gamma_{C}\right)^{\prime}$,

$$
\left\|\psi-\pi_{h}^{C} \psi\right\|_{H^{\frac{1}{2}}\left(\Gamma_{C}\right)^{\prime}} \leq C h^{\frac{1}{2}-\mu}\|\psi\|_{H^{\mu}\left(\Gamma_{C}\right)^{\prime}} .
$$

We also need to use the operator $\pi_{h}^{\partial \Omega}$ constructed in the same way on the whole boundary $\partial \Omega$ and, therefore, satisfying similar approximation estimates with respect to the dual Sobolev norms. Going back to the operator $\mathcal{J}_{h}$, let us introduce the trace space on $\Gamma_{C}$ as

$$
W_{h}\left(\Gamma_{C}\right)=\left\{\psi_{h} \in \mathcal{C}\left(\bar{\Gamma}_{C}\right), \quad \exists v_{h} \in X_{h}(\Omega), \quad \psi_{h \mid \Gamma_{C}}=v_{h \mid \Gamma_{C}}\right\}
$$

and define the one-dimensional interpolation operator $j_{h}: \mathcal{C}\left(\bar{\Gamma}_{C}\right) \rightarrow W_{h}\left(\Gamma_{C}\right)$ to be, $\forall \psi \in \mathcal{C}\left(\bar{\Gamma}_{C}\right)$,

$$
\begin{gathered}
\left(j_{h} \psi\right)\left(\boldsymbol{x}_{i}^{C}\right)=\psi\left(\boldsymbol{x}_{i}^{C}\right), \quad \forall i\left(0 \leq i \leq i^{*}\right), \\
\int_{t_{i}}\left(\psi-j_{h} \psi\right) d \Gamma=0, \quad \forall i\left(0 \leq i \leq i^{*}-1\right) .
\end{gathered}
$$


Then, it is straightforward that for any $v \in \mathcal{C}(\bar{\Omega})$ we have $\left(\mathcal{J}_{h} v\right)_{\mid \Gamma_{C}}=j_{h}\left(v_{\mid \Gamma_{C}}\right)$. As a consequence we have the following: $\forall \psi \in H^{\nu}\left(\Gamma_{C}\right)\left(\frac{1}{2}<\nu \leq 3\right)$,

$$
\left\|\psi-j_{h} \psi\right\|_{L^{2}\left(\Gamma_{C}\right)} \leq C h^{\nu}\|\psi\|_{H^{\nu}\left(\Gamma_{C}\right)} .
$$

Lemma 3.1. For any $\left.\nu \in] \frac{1}{2}, 3\right]$ and any $\mu \in[0,1]$, we have, $\forall \psi \in H^{\nu}\left(\Gamma_{C}\right)$,

$$
\left\|\psi-j_{h} \psi\right\|_{H^{\mu}\left(\Gamma_{C}\right)^{\prime}} \leq C h^{\nu+\mu}\|\psi\|_{H^{\nu}\left(\Gamma_{C}\right)}
$$

Proof. The proof is carried out for $\mu=1$; the case $\mu \in[0,1[$ is handled in the same way. We use the Aubin-Nitsche duality

$$
\left\|\psi-j_{h} \psi\right\|_{H^{1}\left(\Gamma_{C}\right)^{\prime}}=\sup _{\chi \in H^{1}\left(\Gamma_{C}\right)} \frac{1}{\|\chi\|_{H^{1}\left(\Gamma_{C}\right)}} \int_{\Gamma_{C}}\left(\psi-j_{h} \psi\right) \chi d \Gamma .
$$

We have

$$
\begin{aligned}
\int_{\Gamma_{C}}\left(\psi-j_{h} \psi\right) \chi d \Gamma & =\int_{\Gamma_{C}}\left(\psi-j_{h} \psi\right)\left(\chi-\pi_{h}^{C} \chi\right) d \Gamma \\
& \leq\left\|\psi-j_{h} \psi\right\|_{L^{2}\left(\Gamma_{C}\right)}\left\|\chi-\pi_{h}^{C} \chi\right\|_{L^{2}\left(\Gamma_{C}\right)}
\end{aligned}
$$

Using (3.3) with $\mu=0$ and (3.5) leads to

$$
\int_{\Gamma_{C}}\left(\psi-j_{h} \psi\right) \chi d \Gamma \leq C h^{\nu+1}\|\psi\|_{H^{\nu}\left(\Gamma_{C}\right)}\|\chi\|_{H^{1}\left(\Gamma_{C}\right)} .
$$

Thus the proof.

We are in position to define and study the finite element problem issuing from (2.5), in a variational inequality formulation: find $u_{h} \in K_{h}(\Omega)$ such that

$$
a\left(u_{h}, v_{h}-u_{h}\right) \geq L\left(v_{h}-u_{h}\right), \quad \forall v_{h} \in K_{h}(\Omega) .
$$

The set $K_{h}(\Omega)$ is an external approximation of $K(\Omega)$, i.e., $K_{h}(\Omega) \not \subset K(\Omega)$; the discretization is then nonconforming. Nevertheless, proving that the discrete problem (3.6) has only one solution $u_{h} \in K_{h}(\Omega)$ is an easy matter from Stampacchia's Theorem.

\section{NUMERICAL ANALYSIS}

We restrict ourselves to the Signorini solution that belongs to $H^{\nu}(\Omega)$ with $\nu \leq \frac{5}{2}$. As indicated in Remark 2.2, this is in general the effective regularity expected by the theory in the vicinity of $\Gamma_{C}$ (see $[22)$. We have the following error estimate results.

Theorem 4.1. Let $u \in K(\Omega)$ be the solution of the variational Signorini problem (2.5).

i. Assume $u \in H^{\nu}(\Omega)$ with $1<\nu \leq \frac{3}{2}$ and $g \in H^{\frac{3}{2}-\nu}\left(\Gamma_{g}\right)^{\prime}$. Then, the discrete solution $u_{h} \in K_{h}(\Omega)$ is such that

$$
\left\|u-u_{h}\right\|_{H^{1}(\Omega)} \leq C h^{\nu-1}\left(\|u\|_{H^{\nu}(\Omega)}+\|f\|_{L^{2}(\Omega)}+\|g\|_{H^{\frac{3}{2}-\nu}\left(\Gamma_{g}\right)^{\prime}}\right) .
$$

ii. Assume $u \in H^{\nu}(\Omega)$ with $2<\nu \leq \frac{5}{2}$. Then, the discrete solution $u_{h} \in K_{h}(\Omega)$ is such that

$$
\left\|u-u_{h}\right\|_{H^{1}(\Omega)} \leq C h^{\nu-1}\|u\|_{H^{\nu}(\Omega)} .
$$


Theorem 4.2. Let $u \in K(\Omega)$ be the solution of the variational Signorini problem (2.5).

i. Assume that $u \in H^{\nu}(\Omega)$ with $\frac{3}{2}<\nu<2$ and that the number of points in $\Gamma_{C}$, where the constraint changes from binding to nonbinding, is finite. Then, the discrete solution $u_{h} \in K_{h}(\Omega)$ is such that

$$
\left\|u-u_{h}\right\|_{H^{1}(\Omega)} \leq C h^{\nu-1}\|u\|_{H^{\nu}(\Omega)} .
$$

ii. Assume that $u \in H^{2}(\Omega)$ and that the number of points in $\Gamma_{C}$, where the constraint changes from binding to nonbinding, is finite. Then, the discrete solution $u_{h} \in K_{h}(\Omega)$ is such that

$$
\left\|u-u_{h}\right\|_{H^{1}(\Omega)} \leq C h|\log h|^{\frac{1}{4}}\|u\|_{H^{2}(\Omega)} .
$$

Remark 4.3. The results provided by Theorem 4.2 are somehow surprising compared to those given in Theorem 4.1. In view of the optimality attained for $\left.\left.\left.\nu \in] 1, \frac{3}{2}\right] \cup\right] 2, \frac{5}{2}\right]$, without any additional assumption on $u$, we expected to observe similar performances of our method for $\left.\nu \in] \frac{3}{2}, 2\right]$. Unfortunately, the tools developed here fail to produce the desired optimality without assuming that the number of points in $\Gamma_{C}$ where the constraint changes from binding to nonbinding is finite, even thought this working hypothesis, which first appeared in [8] and has since been used in many papers (see [16], 6]), seems to be currently satisfied in particular in solid mechanics. Nevertheless, our belief is that the convergence rate would be also optimal in more general situations and that the problem would be only technical.

Remark 4.4. Of course the regularity exponent $\nu$ on the whole domain should be lower than $\frac{3}{2}$, because of the Dirichlet-Neumann singularities generated around $\left\{\boldsymbol{c}_{1}, \boldsymbol{c}_{2}\right\}$. However, our goal is only to focus on the approximation behavior around $\Gamma_{C}$, so we choose to assume that they are not effective (or in an equivalent way the corresponding singular coefficient is switched-off), which, in view of Remark 2.2 makes the assumptions of Theorem 4.1 and Theorem 4.2 very reasonable. Anyhow, in practice it is possible to reduce the impact of these kind of singularities by resorting to meshes of a particular shape (geometrical or radial meshes) around the Dirichlet-Neumann singular points or by using the algorithm of Strang and Fix (see [23]).

Deriving an estimate of the error $\left(u-u_{h}\right)$ from the exact Signorini solution by our nonconforming quadratic finite element approximation is based on an adaptation of Falk's Lemma (see [12, 44).

Lemma 4.5. Let $u \in K(\Omega)$ be the solution of the variational Signorini inequality $(2.5)$, and $u_{h} \in K_{h}(\Omega)$ the solution of the discrete variational inequality (3.6). Then

$$
\begin{aligned}
& \left\|u-u_{h}\right\|_{H^{1}(\Omega)}^{2} \\
& \leq C \sum_{v_{h} \in K_{h}(\Omega)}\left(\left\|u-v_{h}\right\|_{H^{1}(\Omega)}^{2}+\left\langle\frac{\partial u}{\partial \boldsymbol{n}}, v_{h}-u\right\rangle_{\frac{1}{2}, \partial \Omega}-\left\langle g, v_{h}-u\right\rangle_{\frac{1}{2}, \Gamma_{g}}\right) \\
& \left.\quad+\inf _{v \in K(\Omega)}\left(\left\langle\frac{\partial u}{\partial \boldsymbol{n}}, v-u_{h}\right\rangle_{\frac{1}{2}, \partial \Omega}-\left\langle g, v-u_{h}\right\rangle_{\frac{1}{2}, \Gamma_{g}}\right)\right] .
\end{aligned}
$$

Remark 4.6. The first infimum of the bound given in (4.1) is the approximation error, and the integral term involved there is specifically generated by the discretization of variational inequalities. The last infimum is the consistency error; it is the "variational crime" and is due to the nonconformity of the approximation. 
Before giving the proof of both theorems let us bound separately the approximation and the consistency errors. We start by the approximation error.

Lemma 4.7. Let $u \in K(\Omega)$ be the solution of the variational Signorini problem (2.5). Assume $u \in H^{\nu}(\Omega)$ with $1<\nu \leq \frac{3}{2}$ and $g \in H^{\frac{3}{2}-\nu}\left(\Gamma_{g}\right)^{\prime}$. Then

$$
\begin{aligned}
\inf _{v_{h} \in K_{h}(\Omega)} & \left(\left\|u-v_{h}\right\|_{H^{1}(\Omega)}^{2}+\left\langle\frac{\partial u}{\partial \boldsymbol{n}}, v_{h}-u\right\rangle_{\frac{1}{2}, \partial \Omega}-\left\langle g, v_{h}-u\right\rangle_{\frac{1}{2}, \Gamma_{g}}\right) \\
& \leq C h^{2(\nu-1)}\|u\|_{H^{\nu}(\Omega)}\left(\|u\|_{H^{\nu}(\Omega)}+\|f\|_{L^{2}(\Omega)}+\|g\|_{H^{\frac{3}{2}-\nu_{(}\left(\Gamma_{g}\right)^{\prime}}}\right) .
\end{aligned}
$$

Proof. Observe that, as $u \in H^{\nu}(\Omega)$ and $-\Delta u(=f) \in L^{2}(\Omega)$, then $\left(\frac{\partial u}{\partial \boldsymbol{n}}\right)_{\mid \partial \Omega} \in$ $H^{\frac{3}{2}-\nu}(\partial \Omega)^{\prime}$ with

$$
\left\|\frac{\partial u}{\partial \boldsymbol{n}}\right\|_{H^{\frac{3}{2}-\nu}(\partial \Omega)^{\prime}} \leq C\left(\|u\|_{H^{\nu}(\Omega)}+\|f\|_{L^{2}(\Omega)}\right) .
$$

Then, choosing $v_{h}=\mathcal{J}_{h} u$, on account of 3.2 it turns out that

$$
\left\|u-\mathcal{J}_{h} u\right\|_{H^{1}(\Omega)}^{2} \leq C h^{2(\nu-1)}\|u\|_{H^{\nu}(\Omega)}^{2} .
$$

The estimate of the first integral term is obtained from (3.2) and (4.2):

$$
\begin{aligned}
\left\langle\frac{\partial u}{\partial \boldsymbol{n}}, \mathcal{J}_{h} u-u\right\rangle_{\frac{1}{2}, \partial \Omega} & \leq\left\|\frac{\partial u}{\partial \boldsymbol{n}}\right\|_{H^{\frac{3}{2}-\nu}(\partial \Omega)^{\prime}}\left\|\mathcal{J}_{h} u-u\right\|_{H^{\frac{3}{2}-\nu}(\partial \Omega)} \\
& \leq C h^{2(\nu-1)}\|u\|_{H^{\nu}(\Omega)}\left(\|u\|_{H^{\nu}(\Omega)}+\|f\|_{L^{2}(\Omega)}\right) .
\end{aligned}
$$

The last integral term is bounded in the following way:

$$
\begin{aligned}
\left\langle g, \mathcal{J}_{h} u-u\right\rangle_{\frac{1}{2}, \Gamma_{g}} & \leq\|g\|_{H^{\frac{3}{2}-\nu}\left(\Gamma_{g}\right)^{\prime}}\left\|\mathcal{J}_{h} u-u\right\|_{H^{\frac{3}{2}-\nu}\left(\Gamma_{g}\right)} \\
& \leq C h^{2(\nu-1)}\|u\|_{H^{\nu}(\Omega)}\|g\|_{H^{\frac{3}{2}-\nu}\left(\Gamma_{g}\right)^{\prime}} .
\end{aligned}
$$

Assembling these estimates yields the proof.

Lemma 4.8. Let $u \in K(\Omega)$ be the solution of the variational Signorini problem (2.5). Assume $u \in H^{\nu}(\Omega)$ with $\frac{3}{2}<\nu \leq \frac{5}{2}$. Then

$$
\begin{aligned}
& \inf _{v_{h} \in K_{h}(\Omega)}\left(\left\|u-v_{h}\right\|_{H^{1}(\Omega)}^{2}+\left\langle\frac{\partial u}{\partial \boldsymbol{n}}, v_{h}-u\right\rangle_{\frac{1}{2}, \partial \Omega}-\left\langle g, v_{h}-u\right\rangle_{\frac{1}{2}, \Gamma_{g}}\right) \\
& \quad \leq C h^{2(\nu-1)}\|u\|_{H^{\nu}(\Omega)}^{2}
\end{aligned}
$$

Proof. Since $\nu>\frac{3}{2}$, the normal derivative $\frac{\partial u}{\partial \boldsymbol{n}} \mid \partial \Omega$ belongs to $L^{2}(\partial \Omega)$, and $g\left(=\frac{\partial u}{\partial \boldsymbol{n}} \mid \Gamma_{g}\right) \in L^{2}\left(\Gamma_{g}\right)$. Then, we can write

$$
\left\langle\frac{\partial u}{\partial \boldsymbol{n}}, v_{h}-u\right\rangle_{\frac{1}{2}, \partial \Omega}-\left\langle g, v_{h}-u\right\rangle_{\frac{1}{2}, \Gamma_{g}}=\int_{\Gamma_{C}} \frac{\partial u}{\partial \boldsymbol{n}}\left(v_{h}-u\right) d \Gamma .
$$

Choosing $v_{h}=\mathcal{J}_{h} u$ and using Lemma 3.1, we get

$$
\begin{gathered}
\int_{\Gamma_{C}} \frac{\partial u}{\partial \boldsymbol{n}}\left(\mathcal{J}_{h} u-u\right) d \Gamma \leq\left\|\frac{\partial u}{\partial \boldsymbol{n}}\right\|_{H^{\nu-\frac{3}{2}}\left(\Gamma_{C}\right)}\left\|u-j_{h}\left(u_{\mid \Gamma_{C}}\right)\right\|_{H^{\nu-\frac{3}{2}}\left(\Gamma_{C}\right)^{\prime}} \\
\leq C h^{2(\nu-1)}\left\|\frac{\partial u}{\partial \boldsymbol{n}}\right\|_{H^{\nu-\frac{3}{2}}\left(\Gamma_{C}\right)}\|u\|_{H^{\nu-\frac{1}{2}}\left(\Gamma_{C}\right)} \leq C h^{2(\nu-1)}\|u\|_{H^{\nu}(\Omega)}^{2}
\end{gathered}
$$

Hence the proof. 
Remark 4.9. For higher regularity of $u, \nu>\frac{5}{2}$, the approximation is not optimal any longer because even though $\left(\frac{\partial u}{\partial \boldsymbol{n}}\right)_{\mid \Gamma_{C}} \in H^{\nu-\frac{3}{2}}\left(\Gamma_{C}\right)$ with $\left(\nu-\frac{3}{2}\right)>1$, the best we can prove is that

$$
\left\|u-\mathcal{J}_{h} u\right\|_{H^{\nu-\frac{3}{2}}\left(\Gamma_{C}\right)^{\prime}} \leq\left\|u-\mathcal{J}_{h} u\right\|_{H^{1}\left(\Gamma_{C}\right)^{\prime}} \leq C h^{\nu+\frac{1}{2}}\|u\|_{H^{\nu}(\Omega)} .
$$

This yields the estimate

$$
\left[\inf _{v_{h} \in K_{h}(\Omega)}\left(\left\|u-v_{h}\right\|_{H^{1}(\Omega)}^{2}+\int_{\Gamma_{C}} \frac{\partial u}{\partial \boldsymbol{n}}\left(v_{h}-u\right) d \Gamma\right)\right]^{\frac{1}{2}} \leq C h^{\nu-1} h^{\frac{5}{4}-\frac{\nu}{4}}\|u\|_{H^{\nu}(\Omega)} .
$$

The worst extra factor $h^{\frac{5}{4}-\frac{\nu}{4}}$ shows up for $\nu=3$, where we are $h^{\frac{1}{4}}$ away from optimality (the convergence rate is of order $h^{\frac{7}{4}}$ instead of $h^{2}$ ). However, the quadratic convergence rate can be recovered under the additional assumption of Theorem 4.2

Now, we are left with the consistency error, the analysis of which introduces more technicalities.

Lemma 4.10. Let $u \in K(\Omega)$ be the solution of the variational Signorini problem (2.5). Assume $u \in H^{\nu}(\Omega)$ with $1<\nu \leq \frac{3}{2}$ and $g \in H^{\frac{3}{2}-\nu}\left(\Gamma_{g}\right)^{\prime}$. Then

$$
\begin{gathered}
\inf _{v \in K(\Omega)}\left(\left\langle\frac{\partial u}{\partial \boldsymbol{n}}, v-u_{h}\right\rangle_{\frac{1}{2}, \partial \Omega}-\left\langle g, v-u_{h}\right\rangle_{\frac{1}{2}, \Gamma_{g}}\right) \leq \\
C\left(h^{\nu-1}\left\|u-u_{h}\right\|_{H^{1}(\Omega)}+h^{2(\nu-1)}\|u\|_{H^{\nu}(\Omega)}\right)\left(\|u\|_{H^{\nu}(\Omega)}+\|f\|_{L^{2}(\Omega)}+\|g\|_{H^{\frac{3}{2}-\nu}\left(\Gamma_{g}\right)^{\prime}}\right) .
\end{gathered}
$$

Proof. Define the piecewise constant function $\psi_{h}=\pi_{h}^{\partial \Omega}\left(\frac{\partial u}{\partial \boldsymbol{n}}\right)$, it is clear that $\psi_{h \mid \Gamma_{C}}$ $\geq 0$. Taking $v=u$, then we have

$$
\begin{gathered}
\left\langle\frac{\partial u}{\partial \boldsymbol{n}}, u-u_{h}\right\rangle_{\frac{1}{2}, \partial \Omega}-\left\langle g, u-u_{h}\right\rangle_{\frac{1}{2}, \Gamma_{g}}=\left\langle\psi_{h}, u-u_{h}\right\rangle_{\frac{1}{2}, \partial \Omega}-\left\langle\psi_{h}, u-u_{h}\right\rangle_{\frac{1}{2}, \Gamma_{g}} \\
+\left\langle\frac{\partial u}{\partial \boldsymbol{n}}-\psi_{h}, u-u_{h}\right\rangle_{\frac{1}{2}, \partial \Omega}-\left\langle g-\psi_{h}, u-u_{h}\right\rangle_{\frac{1}{2}, \Gamma_{g}} .
\end{gathered}
$$

The second term is estimated in the following way:

$$
\begin{aligned}
\left\langle\frac{\partial u}{\partial \boldsymbol{n}}\right. & \left.-\psi_{h}, u-u_{h}\right\rangle_{\frac{1}{2}, \partial \Omega}-\left\langle g-\psi_{h}, u-u_{h}\right\rangle_{\frac{1}{2}, \Gamma_{g}} \\
& \leq\left\|\frac{\partial u}{\partial \boldsymbol{n}}-\psi_{h}\right\|_{H^{\frac{1}{2}}(\partial \Omega)^{\prime}}\left\|u-u_{h}\right\|_{H^{\frac{1}{2}}(\partial \Omega)}+\left\|g-\psi_{h}\right\|_{H^{\frac{1}{2}\left(\Gamma_{g}\right)^{\prime}}}\left\|u-u_{h}\right\|_{H^{\frac{1}{2}\left(\Gamma_{g}\right)}} .
\end{aligned}
$$

Therefore, by (3.4) we derive that

$$
\begin{aligned}
\left\langle\frac{\partial u}{\partial \boldsymbol{n}}-\psi_{h}, u-u_{h}\right\rangle_{\frac{1}{2}, \partial \Omega}-\left\langle g-\psi_{h}, u-u_{h}\right\rangle_{\frac{1}{2}, \Gamma_{g}} & \\
& \leq C h^{\nu-1}\left(\|u\|_{H^{\nu}(\Omega)}+\|f\|_{L^{2}(\Omega)}+\|g\|_{H^{\frac{3}{2}-\nu}\left(\Gamma_{g}\right)^{\prime}}\right)\left\|u-u_{h}\right\|_{H^{1}(\Omega)} .
\end{aligned}
$$

To handle the remaining part of (4.3), notice that

$$
\int_{\Gamma_{C}} u_{h} \psi_{h} d \Gamma=\sum_{i=0}^{i^{*}-1}\left(\int_{t_{i}} u_{h} d \Gamma\right) \psi_{h \mid t_{i}} \geq 0
$$

which yields the following bound:

$$
\left\langle\psi_{h}, u-u_{h}\right\rangle_{\frac{1}{2}, \partial \Omega}-\left\langle\psi_{h}, u-u_{h}\right\rangle_{\frac{1}{2}, \Gamma_{g}}=\int_{\Gamma_{C}}\left(u-u_{h}\right) \psi_{h} d \Gamma \leq \int_{\Gamma_{C}} u \psi_{h} d \Gamma .
$$


Thanks to the boundary conditions on $\Gamma_{u}$ and $\Gamma_{g}$ together with the saturation (2.7), we deduce that

$$
\begin{aligned}
& \left\langle\psi_{h}, u-u_{h}\right\rangle_{\frac{1}{2}, \partial \Omega}-\left\langle\psi_{h}, u-u_{h}\right\rangle_{\frac{1}{2}, \Gamma_{g}} \leq\left\langle\psi_{h}-\frac{\partial u}{\partial \boldsymbol{n}}, u\right\rangle_{\frac{1}{2}, \partial \Omega}-\left\langle\psi_{h}-g, u\right\rangle_{\frac{1}{2}, \Gamma_{g}} \\
& \leq\left\|\frac{\partial u}{\partial \boldsymbol{n}}-\psi_{h}\right\|_{H^{\nu-\frac{1}{2}(\partial \Omega)^{\prime}}}\|u\|_{H^{\nu-\frac{1}{2}}(\partial \Omega)}+\left\|g-\psi_{h}\right\|_{H^{\nu-\frac{1}{2}}\left(\Gamma_{g}\right)^{\prime}}\|u\|_{H^{\nu-\frac{1}{2}}(\partial \Omega)}
\end{aligned}
$$

By another use of estimate (3.4) we obtain

$$
\begin{aligned}
\left\langle\psi_{h}, u-u_{h}\right\rangle_{\frac{1}{2}, \partial \Omega}-\left\langle\psi_{h}, u-u_{h}\right\rangle_{\frac{1}{2}, \Gamma_{g}} & \\
& \leq C h^{2(\nu-1)}\left(\left\|\frac{\partial u}{\partial \boldsymbol{n}}\right\|_{H^{\frac{3}{2}-\nu}(\partial \Omega)^{\prime}}\|u\|_{H^{\nu-\frac{1}{2}}(\partial \Omega)}+\|g\|_{H^{\frac{3}{2}-\nu}\left(\Gamma_{g}\right)^{\prime}}\|u\|_{H^{\nu-\frac{1}{2}}(\partial \Omega)}\right) \\
& \leq C h^{2(\nu-1)}\left(\|u\|_{H^{\nu}(\Omega)}+\|f\|_{L^{2}(\Omega)}+\|g\|_{H^{\frac{3}{2}-\nu}\left(\Gamma_{g}\right)^{\prime}}\right)\|u\|_{H^{\nu}(\Omega)} .
\end{aligned}
$$

Lemma 4.11. Let $u \in K(\Omega)$ be the solution of the variational Signorini problem (2.5). Assume $u \in H^{\nu}(\Omega)$ with $2<\nu \leq \frac{5}{2}$. Then

$$
\begin{aligned}
\inf _{v \in K(\Omega)}\left(\left\langle\frac{\partial u}{\partial \boldsymbol{n}}, v-u_{h}\right\rangle_{\frac{1}{2}, \partial \Omega}\right. & \left.-\left\langle g, v-u_{h}\right\rangle_{\frac{1}{2}, \Gamma_{g}}\right) \\
\leq & C\left(h^{\nu-1}\left\|u-u_{h}\right\|_{H^{1}(\Omega)}+h^{2(\nu-1)}\|u\|_{H^{\nu}(\Omega)}\right)\|u\|_{H^{\nu}(\Omega)} .
\end{aligned}
$$

Proof. Taking $v=u$, and thanks to the regularity of $u$, we have

$$
\inf _{v \in K(\Omega)}\left(\left\langle\frac{\partial u}{\partial \boldsymbol{n}}, v-u_{h}\right\rangle_{\frac{1}{2}, \partial \Omega}-\left\langle g, v-u_{h}\right\rangle_{\frac{1}{2}, \Gamma_{g}}\right) \leq \int_{\Gamma_{C}} \frac{\partial u}{\partial \boldsymbol{n}}\left(u-u_{h}\right) d \Gamma .
$$

Setting $\psi_{h}=\pi_{h}^{C}\left(\frac{\partial u}{\partial \boldsymbol{n}}\right) \geq 0$, we get

$$
\begin{aligned}
\int_{\Gamma_{C}} \frac{\partial u}{\partial \boldsymbol{n}}\left(u-u_{h}\right) d \Gamma & =\int_{\Gamma_{C}}\left(\frac{\partial u}{\partial \boldsymbol{n}}-\psi_{h}\right)\left(u-u_{h}\right) d \Gamma+\int_{\Gamma_{C}} \psi_{h}\left(u-u_{h}\right) d \Gamma \\
& \leq \int_{\Gamma_{C}}\left(\frac{\partial u}{\partial \boldsymbol{n}}-\psi_{h}\right)\left(u-u_{h}\right) d \Gamma+\int_{\Gamma_{C}} \psi_{h} u d \Gamma
\end{aligned}
$$

The first part of the bound is handled in a standard way:

$$
\int_{\Gamma_{C}}\left(\frac{\partial u}{\partial \boldsymbol{n}}-\psi_{h}\right)\left(u-u_{h}\right) d \Gamma \leq C h^{\nu-1}\left\|u-u_{h}\right\|_{H^{1}(\Omega)}\|u\|_{H^{\nu}(\Omega)} .
$$

In order to work out the second term, let us define $\chi_{h}=\pi_{h}^{C} u \geq 0$. In view of the saturation $\left(u \frac{\partial u}{\partial \boldsymbol{n}}\right)_{\mid \Gamma_{C}}=0$ we get

$$
\int_{\Gamma_{C}} \psi_{h} u d \Gamma=\int_{\Gamma_{C}}\left(\psi_{h}-\frac{\partial u}{\partial \boldsymbol{n}}\right)\left(u-\chi_{h}\right) d \Gamma=\sum_{i=0}^{i^{*}-1} \int_{t_{i}}\left(\psi_{h}-\frac{\partial u}{\partial \boldsymbol{n}}\right)\left(u-\chi_{h}\right) d \Gamma
$$

The sum can be restricted to the set $I$ of indices $i$ for which $u$ vanishes at least once in $t_{i}$. Indeed, if $u_{\mid t_{i}}>0$, then $\frac{\partial u}{\partial \boldsymbol{n} \mid t_{i}}=0$. This yields $\psi_{h \mid t_{i}}=0$, and therefore $\int_{t_{i}} \psi_{h} u d \Gamma=0$. Then

$$
\begin{aligned}
\int_{\Gamma_{C}} \psi_{h} u d \Gamma & \leq \sum_{i \in I}\left\|\psi_{h}-\frac{\partial u}{\partial \boldsymbol{n}}\right\|_{L^{2}\left(t_{i}\right)}\left\|u-\chi_{h}\right\|_{L^{2}\left(t_{i}\right)} \\
& \leq \sum_{i \in I} C h_{i}^{\nu-\frac{3}{2}}\left\|\frac{\partial u}{\partial \boldsymbol{n}}\right\|_{H^{\nu-\frac{3}{2}\left(t_{i}\right)}} h_{i}|u|_{H^{1}\left(t_{i}\right)} .
\end{aligned}
$$


It remains to estimate the semi-norm $|u|_{H^{1}\left(t_{i}\right)}$. Because for any $i \in I, u_{\mid t_{i}} \geq 0$ and $u$ vanishes at least for one point $\tilde{\boldsymbol{x}}_{i}$, we necessarily have $u^{\prime}\left(\tilde{\boldsymbol{x}}_{i}\right)=0$ (the symbol $'$ stands for the tangential derivative of $u$ along $\Gamma_{C}$ ). This makes sense because $u_{\mid \Gamma_{C}} \in \mathcal{C}^{1, \nu-2}\left(\Gamma_{C}\right)$. Applying Lemma 8.1 to $u^{\prime}$ yields

$$
|u|_{H^{1}\left(t_{i}\right)}=\left\|u^{\prime}\right\|_{L^{2}\left(t_{i}\right)} \leq C h_{i}^{\nu-\frac{3}{2}}\left|u^{\prime}\right|_{H^{\nu-\frac{3}{2}}\left(t_{i}\right)},
$$

so that, going back to (4.4), we obtain

$$
\begin{aligned}
\int_{\Gamma_{C}} \psi_{h} u d \Gamma & \leq \sum_{i \in I} C h_{i}^{\nu-\frac{3}{2}}\left\|\frac{\partial u}{\partial \boldsymbol{n}}\right\|_{H^{\nu-\frac{3}{2}}\left(t_{i}\right)} h_{i}^{\nu-\frac{1}{2}}\left|u^{\prime}\right|_{H^{\nu-\frac{3}{2}}\left(t_{i}\right)} \\
& \leq C h^{2(\nu-1)}\left(\sum_{i \in I}\left\|\frac{\partial u}{\partial \boldsymbol{n}}\right\|_{H^{\nu-\frac{3}{2}}\left(t_{i}\right)}^{2}\right)^{\frac{1}{2}}\left(\sum_{i \in I}\left|u^{\prime}\right|_{H^{\nu-\frac{3}{2}}\left(t_{i}\right)}^{2}\right)^{\frac{1}{2}} \\
& \leq C h^{2(\nu-1)}\left\|\frac{\partial u}{\partial \boldsymbol{n}}\right\|_{H^{\nu-\frac{3}{2}}\left(\Gamma_{C}\right)}\left\|u^{\prime}\right\|_{H^{\nu-\frac{3}{2}}\left(\Gamma_{C}\right)} \leq C h^{2(\nu-1)}\|u\|_{H^{\nu}(\Omega)}^{2} .
\end{aligned}
$$

The proof is finished.

Proof of of Theorem 4.1. Putting together Lemma 4.8 and Lemma 4.11yields

$$
\left\|u-u_{h}\right\|_{H^{1}(\Omega)}^{2} \leq C h^{\nu-1}\left\|u-u_{h}\right\|_{H^{1}(\Omega)}\|u\|_{H^{\nu}(\Omega)}+h^{2(\nu-1)}\|u\|_{H^{\nu}(\Omega)}^{2}
$$

from which point $i$. of the theorem follows. Point $i$. is proven in the same manner using Lemmas 4.4 and 4.6.

Proving Theorem 4.2 requires two more technical lemmas, also dedicated to the analysis of the consistency error.

Lemma 4.12. Let $u \in K(\Omega)$ be the solution of the variational Signorini problem (2.5). Assume that $u \in H^{\nu}(\Omega)$ with $\frac{3}{2}<\nu<2$, and that the number of points in $\Gamma_{C}$, where the constraint changes from binding to nonbinding is finite. Then

$$
\begin{aligned}
\inf _{v \in K(\Omega)}\left(\left\langle\frac{\partial u}{\partial \boldsymbol{n}}, v-u_{h}\right\rangle_{\frac{1}{2}, \partial \Omega}\right. & \left.-\left\langle g, v-u_{h}\right\rangle_{\frac{1}{2}, \Gamma_{g}}\right) \\
\leq & C\left(h^{\nu-1}\left\|u-u_{h}\right\|_{H^{1}(\Omega)}+h^{2(\nu-1)}\|u\|_{H^{\nu}(\Omega)}\right)\|u\|_{H^{\nu}(\Omega)} .
\end{aligned}
$$

Proof. Denote by $I$ the set of indices $i\left(0 \leq i \leq i^{*}-1\right)$ corresponding to the segments $t_{i}$ containing at least one point where the constraint changes from binding to nonbinding. The cardinality of $I$ is bounded uniformly in $h$. It is straightforward that in each $t_{i}, i \notin I$, the product $\left(\psi_{h} u\right)_{\mid t_{i}}=0$, because either $u_{\mid t_{i}}=0$ or $u_{\mid t_{i}}>0$; then $\left(\frac{\partial u}{\partial n}\right)_{\mid t_{i}}=0$ and $\psi_{h \mid t_{i}}=0$. Proceeding as in the proof of the previous lemma, the term that remains to bound is

$$
\begin{aligned}
\int_{\Gamma_{C}} \psi_{h} u d \Gamma & =\int_{\Gamma_{C}}\left(\psi_{h}-\frac{\partial u}{\partial \boldsymbol{n}}\right) u d \Gamma \\
\leq \sum_{i \in I}\left\|\psi_{h}-\frac{\partial u}{\partial \boldsymbol{n}}\right\|_{L^{2}\left(t_{i}\right)}\|u\|_{L^{2}\left(t_{i}\right)} & \leq \sum_{i \in I} C h_{i}^{\nu-\frac{3}{2}}\left\|\frac{\partial u}{\partial \boldsymbol{n}}\right\|_{H^{\nu-\frac{3}{2}\left(t_{i}\right)}} h_{i}^{\frac{1}{2}}\|u\|_{L^{\infty}\left(t_{i}\right)} .
\end{aligned}
$$

By Sobolev-Morrey we have the continuous embedding $H^{\nu-\frac{1}{2}}\left(\Gamma_{C}\right) \subset \mathcal{C}^{0, \nu-1}\left(\Gamma_{C}\right)$. Observing that $u$ vanishes at least once in $t_{i}, i \in I$, we see that

$$
\|u\|_{L^{\infty}\left(t_{i}\right)} \leq h^{\nu-1} \sup _{\boldsymbol{x}, \boldsymbol{y} \in t_{i}} \frac{|u(\boldsymbol{x})-u(\boldsymbol{y})|}{|\boldsymbol{x}-\boldsymbol{y}|^{\nu-1}} \leq h^{\nu-1}\|u\|_{\mathcal{C}^{0, \nu-1}\left(\Gamma_{C}\right)} .
$$


Inserting this in (4.5) and observing that card $I$ is finite yield

$$
\begin{aligned}
\int_{\Gamma_{C}} \psi_{h} u d \Gamma & \leq \sum_{i \in I} C h_{i}^{\nu-\frac{3}{2}}\left\|\frac{\partial u}{\partial \boldsymbol{n}}\right\|_{H^{\nu-\frac{3}{2}}\left(t_{i}\right)} h_{i}^{\nu-\frac{1}{2}}\|u\|_{\mathcal{C}^{0, \nu-1}\left(\Gamma_{C}\right)} \\
& \leq C h^{2(\nu-1)}\left\|\frac{\partial u}{\partial \boldsymbol{n}}\right\|_{H^{\nu-\frac{3}{2}}\left(\Gamma_{C}\right)}\|u\|_{\mathcal{C}^{0, \nu-1}\left(\Gamma_{C}\right)} \leq C h^{2(\nu-1)}\|u\|_{H^{\nu}(\Omega)}^{2} .
\end{aligned}
$$

This ends the proof.

Lemma 4.13. Let $u \in K(\Omega)$ be the solution of the variational Signorini problem (2.5). Assume that $u \in H^{2}(\Omega)$, and that the number of points in $\Gamma_{C}$ where the constraint changes from binding to nonbinding is finite. Then

$$
\begin{aligned}
\inf _{v \in K(\Omega)}\left(\left\langle\frac{\partial u}{\partial \boldsymbol{n}}, v-u_{h}\right\rangle_{\frac{1}{2}, \partial \Omega}\right. & \left.-\left\langle g, v-u_{h}\right\rangle_{\frac{1}{2}, \Gamma_{g}}\right) \\
\leq & C\left(h\left\|u-u_{h}\right\|_{H^{1}(\Omega)}+h^{2}|\log h|^{\frac{1}{2}}\|u\|_{H^{2}(\Omega)}\right)\|u\|_{H^{2}(\Omega)} .
\end{aligned}
$$

Proof. First recall that for any $\alpha \in\left[0,1\right.$ [ the embedding $H^{\frac{3}{2}}\left(\Gamma_{C}\right) \subset \mathcal{C}^{0, \alpha}\left(\Gamma_{C}\right)$ is continuous and there exists a constant $C>0$ independent of $\alpha$ such that (see [3], Lemma A.2), $\forall \psi \in H^{\frac{3}{2}}\left(\Gamma_{C}\right)$,

$$
\|\psi\|_{\mathcal{C}^{0, \alpha}\left(\Gamma_{C}\right)} \leq C \frac{1}{\sqrt{1-\alpha}}\|\psi\|_{H^{\frac{3}{2}}\left(\Gamma_{C}\right)}
$$

As in the proof of Lemma 4.12, we obtain

$$
\begin{aligned}
\int_{\Gamma_{C}} \psi_{h} u d \Gamma & \leq \sum_{i \in I} C h_{i}^{\frac{1}{2}}\left\|\frac{\partial u}{\partial \boldsymbol{n}}\right\|_{H^{\frac{1}{2}}\left(t_{i}\right)} h_{i}^{\frac{1}{2}+\alpha}\|u\|_{\mathcal{C}^{0, \alpha}\left(\Gamma_{C}\right)} \\
& \leq C h^{1+\alpha}\left\|\frac{\partial u}{\partial \boldsymbol{n}}\right\|_{H^{\frac{1}{2}}\left(\Gamma_{C}\right)}\|u\|_{\mathcal{C}^{0, \alpha}\left(\Gamma_{C}\right)} \\
& \leq C h^{2} \frac{h^{\alpha-1}}{\sqrt{1-\alpha}}\left\|\frac{\partial u}{\partial \boldsymbol{n}}\right\|_{H^{\frac{1}{2}\left(\Gamma_{C}\right)}}\|u\|_{H^{\frac{3}{2}}\left(\Gamma_{C}\right)} .
\end{aligned}
$$

Choosing $\alpha=1-\frac{1}{|\log h|}$ achieves the result.

Proof of Theorem 4.2. Putting together Lemma 4.8 and Lemma 4.12 gives point $i$. of the theorem, while point ii. is obtained from Lemma 4.8 and Lemma 4.13 .

\section{Another QUadratic Finite ElEMENTS Discretization}

An alternative to the numerical model of the contact condition presented in the previous section consists in enforcing the nonnegativity of the Lagrange degrees of freedom of the discrete solution that are located on the contact region $\Gamma_{C}$, i.e., $u_{h}\left(\boldsymbol{x}_{i}^{C}\right) \geq 0\left(1 \leq i \leq i^{*}\right)$ and $u_{h}\left(\boldsymbol{x}_{i+\frac{1}{2}}^{C}\right) \geq 0\left(1 \leq i \leq i^{*}-1\right)$. This choice seems more appropriate when we are interested in checking the condition $u_{\mid \Gamma_{C}} \geq 0$ rather than $\left.\frac{\partial u}{\partial \boldsymbol{n}}\right|_{\Gamma_{C}} \geq 0$ (for which the first model appears well adapted). The closed convex cone of work is defined to be

$$
\begin{aligned}
& \tilde{K}_{h}(\Omega)=\left\{v_{h} \in X_{h}(\Omega), \quad v_{h}\left(\boldsymbol{x}_{i}^{C}\right) \geq 0, \quad \forall i\left(0 \leq i \leq i^{*}\right)\right. \\
& \left.v_{h}\left(\boldsymbol{x}_{i+\frac{1}{2}}^{C}\right) \geq 0, \quad \forall i\left(0 \leq i \leq i^{*}-1\right)\right\} .
\end{aligned}
$$


The discrete variational inequality is expressed in the same line as for the first method and consists of: find $\tilde{u}_{h} \in \tilde{K}_{h}(\Omega)$ such that

$$
a\left(\tilde{u}_{h}, v_{h}-\tilde{u}_{h}\right) \geq L\left(v_{h}-\tilde{u}_{h}\right), \quad \forall v_{h} \in \tilde{K}_{h}(\Omega) .
$$

Clearly this method is also nonconforming, because $\tilde{K}_{h}(\Omega) \not \subset K(\Omega)$. Using again Stampacchia's Theorem, we deduce the well posedness of this problem with a stability result; the approximated solution is continuous with respect to the data. The reliability of the approximation is summarized in two theorems.

Theorem 5.1. Let $u \in K(\Omega)$ be the solution of the variational Signorini problem (2.5).

i. Assume $u \in H^{\nu}(\Omega)$ with $1<\nu \leq \frac{3}{2}$, and $g \in H^{\frac{3}{2}-\nu}\left(\Gamma_{g}\right)^{\prime}$. Then, the discrete solution $\tilde{u}_{h} \in \tilde{K}_{h}(\Omega)$ of problem (5.1) is such that

$$
\left\|u-\tilde{u}_{h}\right\|_{H^{1}(\Omega)} \leq C h^{\nu-1}\left(\|u\|_{H^{\nu}(\Omega)}+\|f\|_{L^{2}(\Omega)}+\|g\|_{H^{\frac{3}{2}-\nu}\left(\Gamma_{g}\right)^{\prime}}\right) .
$$

ii. Assume $u \in H^{\nu}(\Omega)$ with $2<\nu \leq \frac{5}{2}$. Then, the discrete solution $\tilde{u}_{h} \in \tilde{K}_{h}(\Omega)$ of problem (5.1) is such that

$$
\left\|u-\tilde{u}_{h}\right\|_{H^{1}(\Omega)} \leq C h^{\nu-1}\|u\|_{H^{\nu}(\Omega)} .
$$

Theorem 5.2. Let $u \in K(\Omega)$ be the solution of the variational Signorini problem (2.5).

i. Assume that $u \in H^{\nu}(\Omega)$ with $\frac{3}{2}<\nu<2$, and that the number of points in $\Gamma_{C}$ where the constraint changes from binding to nonbinding is finite. Then the discrete solution $\tilde{u}_{h} \in \tilde{K}_{h}(\Omega)$ of problem (5.1) is such that

$$
\left\|u-\tilde{u}_{h}\right\|_{H^{1}(\Omega)} \leq C h^{\nu-1}\|u\|_{H^{\nu}(\Omega)} .
$$

ii. Assume that $u \in H^{2}(\Omega)$, and that the number of points in $\Gamma_{C}$ where the constraint changes from binding to nonbinding is finite. Then the discrete solution $\tilde{u}_{h} \in \tilde{K}_{h}(\Omega)$ of problem (5.1) is such that

$$
\left\|u-\tilde{u}_{h}\right\|_{H^{1}(\Omega)} \leq C h|\log h|^{\frac{1}{4}}\|u\|_{H^{2}(\Omega)} .
$$

Before starting the numerical analysis of this method, which is also based on Falk's Lemma 4.3, replacing $K_{h}(\Omega)$ by $\tilde{K}_{h}(\Omega)$, let us make the following observation. By Simpson's quadrature formula we have, $\forall v_{h} \in \tilde{K}_{h}(\Omega)$,

$$
\int_{t_{i}} v_{h} d \Gamma \geq 0, \quad \forall i\left(0 \leq i \leq i^{*}-1\right) .
$$

This implies that $\tilde{K}_{h}(\Omega) \subset K_{h}(\Omega)$, and the principal consequence is that the analysis of the consistency error induced by $\tilde{K}_{h}(\Omega)$ can be made exactly as for $K_{h}(\Omega)$, and the convergence rate will be the same as those provided by Lemmas 4.104 .13 The only remaining point is to exhibit an estimate of the approximation error, which turns out to be more technical than for the first method and is the subject of the following lemmas. 
Lemma 5.3. Let $u \in K(\Omega)$ be the solution of the variational Signorini problem (2.5). Assume $u \in H^{\nu}(\Omega)$ with $1<\nu \leq \frac{3}{2}$, and $g \in H^{\frac{3}{2}-\nu}\left(\Gamma_{g}\right)^{\prime}$. Then

$$
\begin{aligned}
& \inf _{v_{h} \in \tilde{K}_{h}(\Omega)}\left(\left\|u-v_{h}\right\|_{H^{1}(\Omega)}^{2}+\left\langle\frac{\partial u}{\partial \boldsymbol{n}}, v_{h}-u\right\rangle_{\frac{1}{2}, \partial \Omega}-\left\langle g, v_{h}-u\right\rangle_{\frac{1}{2}, \Gamma_{g}}\right) \\
& \leq C h^{2(\nu-1)}\|u\|_{H^{\nu}(\Omega)}\left(\|u\|_{H^{\nu}(\Omega)}+\|f\|_{L^{2}(\Omega)}+\|g\|_{H^{\frac{3}{2}-\nu}\left(\Gamma_{g}\right)^{\prime}}\right) .
\end{aligned}
$$

Proof. Choose $v_{h}=\mathcal{I}_{h} u$ and proceed as in the proof of Lemma 4.7.

Lemma 5.4. Let $u \in K(\Omega)$ be the solution of the variational Signorini problem (2.5). Assume $u \in H^{\nu}(\Omega)$ with $2<\nu \leq \frac{5}{2}$. Then

$$
\begin{aligned}
& \inf _{v_{h} \in \tilde{K}_{h}(\Omega)}\left(\left\|u-v_{h}\right\|_{H^{1}(\Omega)}^{2}+\left\langle\frac{\partial u}{\partial \boldsymbol{n}}, v_{h}-u\right\rangle_{\frac{1}{2}, \partial \Omega}-\left\langle g, v_{h}-u\right\rangle_{\frac{1}{2}, \Gamma_{g}}\right) \\
& \quad \leq C h^{2(\nu-1)}\|u\|_{H^{\nu}(\Omega)}^{2} .
\end{aligned}
$$

Proof. Taking $v_{h}=\mathcal{I}_{h} u$, and thanks to (3.1), we have

$$
\left\|u-v_{h}\right\|_{H^{1}(\Omega)} \leq C h^{\nu-1}\|u\|_{H^{\nu}(\Omega)} .
$$

In order to study the integral term, notice that due to the regularity of $u$ it is reduced to

$$
\begin{aligned}
\left\langle\frac{\partial u}{\partial \boldsymbol{n}}, v_{h}-u\right\rangle_{\frac{1}{2}, \partial \Omega} & -\left\langle g, v_{h}-u\right\rangle_{\frac{1}{2}, \Gamma_{g}}=\int_{\Gamma_{C}} \frac{\partial u}{\partial \boldsymbol{n}}\left(\mathcal{I}_{h} u-u\right) d \Gamma \\
& =\sum_{i=0}^{i^{*}-1} \int_{t_{i}} \frac{\partial u}{\partial \boldsymbol{n}}\left(\mathcal{I}_{h} u-u\right) d \Gamma \leq \sum_{i=0}^{i^{*}-1}\left\|\frac{\partial u}{\partial \boldsymbol{n}}\right\|_{L^{2}\left(t_{i}\right)}\left\|u-\mathcal{I}_{h} u\right\|_{L^{2}\left(t_{i}\right)}
\end{aligned}
$$

The sum can be restricted to the set $I$ of indices $i$ for which $\left(\frac{\partial u}{\partial \boldsymbol{n}}\right)_{\mid \Gamma_{C}} \subset H^{\nu-\frac{3}{2}}\left(\Gamma_{C}\right) \subset$ $\mathcal{C}\left(\Gamma_{C}\right)$ vanishes at least once in $t_{i}$, because if $\frac{\partial u}{\partial \boldsymbol{n}_{\mid t_{i}}}>0$ then $u_{\mid t_{i}}=0$; this yields $\left(\mathcal{I}_{h} u\right)_{\mid t_{i}}=0$. Then

$$
\int_{\Gamma_{C}} \frac{\partial u}{\partial \boldsymbol{n}}\left(\mathcal{I}_{h} u-u\right) d \Gamma \leq C \sum_{i \in I}\left\|\frac{\partial u}{\partial \boldsymbol{n}}\right\|_{L^{2}\left(t_{i}\right)} h_{i}^{\nu-\frac{1}{2}}|u|_{H^{\nu-\frac{1}{2}}\left(t_{i}\right)} .
$$

By Lemma 8.1 applied to $\frac{\partial u}{\partial \boldsymbol{n}}$ (which vanishes at least at one point of $t_{i}$ ) we obtain

$$
\begin{aligned}
\int_{\Gamma_{C}} \frac{\partial u}{\partial \boldsymbol{n}}\left(\mathcal{I}_{h} u-u\right) d \Gamma & \leq \sum_{i \in I} C h_{i}^{\nu-\frac{3}{2}}\left|\frac{\partial u}{\partial \boldsymbol{n}}\right|_{H^{\nu-\frac{3}{2}}\left(t_{i}\right)} h_{i}^{\nu-\frac{1}{2}}|u|_{H^{\nu-\frac{1}{2}}\left(t_{i}\right)} \\
& \leq C h^{2(\nu-1)}\left(\sum_{i \in I}\left|\frac{\partial u}{\partial \boldsymbol{n}}\right|_{H^{\nu-\frac{3}{2}}\left(t_{i}\right)}^{2}\right)^{\frac{1}{2}}\left(\sum_{i \in I}|u|_{H^{\nu-\frac{1}{2}}\left(t_{i}\right)}^{2}\right)^{\frac{1}{2}} \\
& \leq C h^{2(\nu-1)}\left|\frac{\partial u}{\partial \boldsymbol{n}}\right|_{H^{\nu-\frac{3}{2}}\left(\Gamma_{C}\right)}|u|_{H^{\nu-\frac{1}{2}}\left(\Gamma_{C}\right)} \leq C h^{2(\nu-1)}\|u\|_{H^{\nu}(\Omega)}^{2} .
\end{aligned}
$$

The proof is finished.

Proof of Theorem 5.1. We combine Lemmas 4.10 and 5.3 for point $i$ and Lemmas 4.11 and 5.4 to obtain point $i$.

Let us turn to the lemmas necessary for the proof of Theorem 5.2 for which an additional assumption is required on the exact solution. 
Lemma 5.5. Let $u \in K(\Omega)$ be the solution of the variational Signorini problem (2.5). Assume that $u \in H^{\nu}(\Omega)$ with $\frac{3}{2}<\nu<2$, and that the number of points in $\Gamma_{C}$ where the constraint changes from binding to nonbinding is finite. Then

$$
\begin{aligned}
& \inf _{v_{h} \in \tilde{K}_{h}(\Omega)}\left(\left\|u-v_{h}\right\|_{H^{1}(\Omega)}^{2}+\left\langle\frac{\partial u}{\partial \boldsymbol{n}}, v_{h}-u\right\rangle_{\frac{1}{2}, \partial \Omega}-\left\langle g, v_{h}-u\right\rangle_{\frac{1}{2}, \Gamma_{g}}\right) \\
& \quad \leq C h^{2(\nu-1)}\|u\|_{H^{\nu}(\Omega)}^{2} .
\end{aligned}
$$

Proof. We use the arguments developed in ([3], Lemma 2.4). Let $I$ be the set of indices $i\left(0 \leq i \leq i^{*}-1\right)$ such that $t_{i}$ contains at least one point where the constraint changes from binding to nonbinding. In $t_{i}, i \notin I$, the product $\left(\frac{\partial u}{\partial \boldsymbol{n}}\left(u-\mathcal{I}_{h} u\right)\right)_{\mid t_{i}}=0$. Setting $p=(\nu-1)^{-1}$ and $p^{\prime}=(2-\nu)^{-1}$, clearly we have $p, p^{\prime} \geq 1$ and $\frac{1}{p}+\frac{1}{p^{\prime}}=1$. Then, as $\frac{\partial u}{\partial \boldsymbol{n}} \in H^{\nu-\frac{3}{2}}\left(\Gamma_{C}\right)$ and $u \in H^{\nu-\frac{1}{2}}\left(\Gamma_{C}\right)$, invoking the continuous Sobolev embedding (see [1], Theorem 7.48),

$$
H^{\nu-\frac{3}{2}}\left(\Gamma_{C}\right) \subset L^{p^{\prime}}\left(\Gamma_{C}\right), \quad H^{\nu-\frac{1}{2}}\left(\Gamma_{C}\right) \subset L^{p}\left(\Gamma_{C}\right),
$$

we find that $\left(\frac{\partial u}{\partial \boldsymbol{n}}\right)_{\mid \Gamma_{C}} \in L^{p^{\prime}}\left(\Gamma_{C}\right)$ and $u_{\mid \Gamma_{C}} \in L^{p}\left(\Gamma_{C}\right)$. Using the Hölder inequality yields

$$
\begin{aligned}
\int_{\Gamma_{C}} \frac{\partial u}{\partial \boldsymbol{n}}\left(\mathcal{I}_{h} u-u\right) d \Gamma & \leq \sum_{i \in I}\left\|\frac{\partial u}{\partial \boldsymbol{n}}\right\|_{L^{p^{\prime}\left(t_{i}\right)}}\left\|\mathcal{I}_{h} u-u\right\|_{L^{p}\left(t_{i}\right)} \\
& \leq \sum_{i \in I}\left\|\frac{\partial u}{\partial \boldsymbol{n}}\right\|_{L^{p^{\prime}\left(t_{i}\right)}} h_{i}^{\frac{1}{p}}\left\|\mathcal{I}_{h} u-u\right\|_{L^{\infty}\left(t_{i}\right)} .
\end{aligned}
$$

Resorting to the Gagliardo-Nirenberg inequality produces

$$
\left\|\mathcal{I}_{h} u-u\right\|_{L^{\infty}\left(t_{i}\right)} \leq\left\|\mathcal{I}_{h} u-u\right\|_{L^{2}\left(t_{i}\right)}^{\frac{1}{2}}\left\|\mathcal{I}_{h} u-u\right\|_{H^{1}\left(t_{i}\right)}^{\frac{1}{2}} \leq C h_{i}^{\nu-1}|u|_{H^{\nu-\frac{1}{2}\left(t_{i}\right)}} .
$$

Going back to (5.2), and recalling that card $I$ is bounded uniformly in $h$, we write

$$
\int_{\Gamma_{C}} \frac{\partial u}{\partial \boldsymbol{n}}\left(\mathcal{I}_{h} u-u\right) d \Gamma \leq C\left\|\frac{\partial u}{\partial \boldsymbol{n}}\right\|_{L^{p^{\prime}\left(\Gamma_{c}\right)}} \sum_{i \in I} h_{i}^{\frac{1}{p}} h_{i}^{\nu-1}|u|_{H^{\nu-\frac{1}{2}\left(t_{i}\right)}} \leq C h_{i}^{2(\nu-1)}\|u\|_{H^{\nu}(\Omega)}^{2} .
$$

Hence the proof.

Lemma 5.6. Let $u \in K(\Omega)$ be the solution of the variational Signorini problem (2.5). Assume that $u \in H^{2}(\Omega)$, and that the number of points in $\Gamma_{C}$ where the constraint changes from binding to nonbinding is finite. Then

$$
\begin{aligned}
& \inf _{v_{h} \in \tilde{K}_{h}(\Omega)}\left(\left\|u-v_{h}\right\|_{H^{1}(\Omega)}^{2}+\left\langle\frac{\partial u}{\partial \boldsymbol{n}}, v_{h}-u\right\rangle_{\frac{1}{2}, \partial \Omega}-\left\langle g, v_{h}-u\right\rangle_{\frac{1}{2}, \Gamma_{g}}\right) \\
& \quad \leq C h^{2}|\log h|^{\frac{1}{2}}\|u\|_{H^{\nu}(\Omega)}^{2} .
\end{aligned}
$$

Proof. As in the previous lemma, the hardest task is to estimate the integral term. First, recall that for any $p^{\prime} \geq 1$ the embedding $H^{\frac{1}{2}}\left(\Gamma_{C}\right) \subset L^{p^{\prime}}\left(\Gamma_{C}\right)$ is continuous and there exists a constant $C>0$ independent of $p^{\prime}$ such that (see [3], Lemma A.1), $\forall \psi \in H^{\frac{1}{2}}\left(\Gamma_{C}\right)$,

$$
\|\psi\|_{L^{p^{\prime}}\left(\Gamma_{C}\right)} \leq C \sqrt{p^{\prime}}\|\psi\|_{H^{\frac{1}{2}}\left(\Gamma_{C}\right)}
$$


As in the proof of Lemma 5.5. we derive that

$$
\begin{aligned}
\int_{\Gamma_{C}} \frac{\partial u}{\partial \boldsymbol{n}}\left(\mathcal{I}_{h} u-u\right) d \Gamma & \leq C\left\|\frac{\partial u}{\partial \boldsymbol{n}}\right\|_{L^{p^{\prime}}\left(\Gamma_{c}\right)} \sum_{i \in I} h_{i}^{\frac{1}{p}} h_{i}|u|_{H^{\frac{3}{2}}\left(t_{i}\right)} \\
& \leq C h^{2} h^{-\frac{1}{p^{\prime}}}\left\|\frac{\partial u}{\partial \boldsymbol{n}}\right\|_{L^{p^{\prime}\left(\Gamma_{c}\right)}}|u|_{H^{\frac{3}{2}}\left(\Gamma_{C}\right)},
\end{aligned}
$$

where $p$ and $p^{\prime}$ are conjugate real numbers. Applying (5.3) to $\frac{\partial u}{\partial \boldsymbol{n}}$, and since $I$ is finite uniformly with respect to $h$, we get

$$
\int_{\Gamma_{C}} \frac{\partial u}{\partial \boldsymbol{n}}\left(\mathcal{I}_{h} u-u\right) d \Gamma \leq C\left(\sqrt{p^{\prime}} h^{-\frac{1}{p^{\prime}}}\right) h^{2}\left\|\frac{\partial u}{\partial \boldsymbol{n}}\right\|_{H^{\frac{1}{2}}\left(\Gamma_{C}\right)}|u|_{H^{\frac{3}{2}}\left(\Gamma_{C}\right)} .
$$

Taking $p^{\prime}=|\log h|$ completes the proof.

Proof of Theorem 5.2 Put together Lemmas 4.12 and 5.5 for point $i$, and Lemmas 4.13 and 5.6 to obtain point ii.

\section{Conclusion}

The numerical models proposed here, to take into account - in a weak sense - the Signorini nonpenetration condition for a quadratic finite element approximation, provide the expected convergence results for almost all interesting configurations. Using these methods to compute the solution of unilateral contact problems of Signorini type is more accurate than the linear finite element solution.

There are two obvious directions in which this work could be extended. First is the extension of these numerical contact models to three dimensions, for which the technical difficulties are even more increased. The second consists in using the mortar concept introduced in [7, (see also 6], 3], 20, 10, for linear finite elements applied to unilateral contact inequalities) in order to match noncoinciding meshes in the quadratic finite element framework. This approach is of major importance especially for the simulation of unilateral contact between two elastic solids.

\section{Appendix A}

The main purpose of this appendix is to state the nonstandard estimate (3.4) on the piecewise constant interpolation operator. The proof can be found in [5] for any space dimension; it is given here in one dimension to be self contained. For simplicity we work on the reference segment $\Gamma=(0,1)$. Consider the subdivision $\left(x_{i}\right)_{0 \leq i \leq i^{*}}$ ordered increasingly, with $x_{0}=0, x_{i^{*}}=1$. Define $t_{i}=\left(x_{i}, x_{i+1}\right)$, and let $h_{i}=\left|t_{i}\right|=\left|x_{i+1}-x_{i}\right|$ be the length of $t_{i}$; we assume that $h_{i} \leq h(0 \leq i \leq$ $\left.i^{*}-1\right)$. The finite element space $M_{h}(\Gamma)$ involves the piecewise constant functions, i.e., $\psi_{h} \in M_{h}(\Gamma)$ means that $\psi_{h \mid t_{i}} \in \mathcal{P}_{0}\left(t_{i}\right)\left(0 \leq i \leq i^{*}-1\right)$. The $L^{2}$-orthogonal projection $\pi_{h}$ on $M_{h}(\Gamma)$ is then characterized by

$$
\left(\pi_{h} \psi\right)_{\mid t_{i}}=\frac{1}{\left|t_{i}\right|} \int_{t_{i}} \psi d \Gamma, \quad \forall i\left(0 \leq i \leq i^{*}-1\right) .
$$

The approximation error (3.3) for $\pi_{h}$ is standard and is obtained by the AubinNitsche argument. Before stating the desired results let us recall the Hardy inequality. If $\mu \in\left[0, \frac{1}{2}\left[\right.\right.$, then we have, $\forall \psi \in H^{\mu}(0,1)$,

$$
\left(\int_{(0,1)} \frac{\psi(x)^{2}}{x^{2 \mu}} d x\right)^{\frac{1}{2}} \leq c\|\psi\|_{H^{\mu}(0,1)} .
$$


We need the following intermediary lemma.

Lemma 7.1. Let $\mu \in\left[0, \frac{1}{2}\left[\right.\right.$ and $\nu \in[\mu, 1]$. Then $\forall \psi \in H^{\nu}(0,1)$,

$$
\left\|\psi-\pi_{h} \psi\right\|_{H^{\mu}(\Gamma)} \leq C h^{\nu-\mu}\|\psi\|_{H^{\nu}(\Gamma)} .
$$

Proof. Set $\psi_{h}=\pi_{h} \psi$. We have to bound

$$
\begin{aligned}
\left|\psi-\pi_{h} \psi\right|_{H^{\mu}(\Gamma)}^{2}= & \int_{\Gamma} \int_{\Gamma} \frac{\left[\left(\psi-\psi_{h}\right)(x)-\left(\psi-\psi_{h}\right)(y)\right]^{2}}{|x-y|^{1+2 \mu}} d x d y \\
= & \sum_{i=0}^{i^{*}-1} \int_{t_{i}} \int_{t_{i}} \frac{[\psi(x)-\psi(y)]^{2}}{|x-y|^{1+2 \mu}} d x d y \\
& +\sum_{i=0}^{i^{*}-1} \sum_{j \neq i} \int_{t_{i}} \int_{t_{j}} \frac{\left[\left(\psi-\psi_{h}\right)(x)-\left(\psi-\psi_{h}\right)(y)\right]^{2}}{|x-y|^{1+2 \mu}} d x d y
\end{aligned}
$$

It is straightforward that

$$
\begin{aligned}
& \sum_{i=0}^{i^{*}-1} \int_{t_{i}} \int_{t_{i}} \frac{[\psi(x)-\psi(y)]^{2}}{|x-y|^{1+2 \mu}} d x d y \\
& \quad \leq h^{2(\nu-\mu)} \sum_{i=0}^{i^{*}-1} \int_{t_{i}} \int_{t_{i}} \frac{[\psi(x)-\psi(y)]^{2}}{|x-y|^{1+2 \nu}} d x d y \leq h^{2(\nu-\mu)}|\psi|_{H^{\nu}(\Gamma)}^{2}
\end{aligned}
$$

The second sum in (17.2) is bounded as follows:

$$
\begin{aligned}
& \sum_{i=0}^{i^{*}-1} \sum_{j \neq i} \int_{t_{i}} \int_{t_{j}} \frac{\left[\left(\psi-\psi_{h}\right)(x)-\left(\psi-\psi_{h}\right)(y)\right]^{2}}{|x-y|^{1+2 \mu}} d x d y \\
& \quad \leq \sum_{i=0}^{i^{*}-1} \sum_{j \neq i} \int_{t_{i}} \int_{t_{j}} \frac{\left[\left(\psi-\psi_{h}\right)(x)\right]^{2}}{|x-y|^{1+2 \mu}} d x d y+\sum_{i=0}^{i^{*}-1} \sum_{j \neq i} \int_{t_{i}} \int_{t_{j}} \frac{\left[\left(\psi-\psi_{h}\right)(y)\right]^{2}}{|x-y|^{1+2 \mu}} d x d y .
\end{aligned}
$$

We only focus on the the first term (the second is worked out exactly in the same way):

$$
\begin{aligned}
& \sum_{i=0}^{i^{*}-1} \sum_{j \neq i} \int_{t_{i}} \int_{t_{j}} \frac{\left[\left(\psi-\psi_{h}\right)(x)\right]^{2}}{|x-y|^{1+2 \mu}} d x d y \\
& \quad=\sum_{i=0}^{i^{*}-1} \int_{t_{i}}\left[\left(\psi-\psi_{h}\right)(x)\right]^{2}\left(\int_{\Gamma \backslash t_{i}} \frac{1}{|x-y|^{1+2 \mu}} d y\right) d x \\
& \quad \leq C \sum_{i=0}^{i^{*}-1} \int_{t_{i}}\left[\left(\psi-\psi_{h}\right)(x)\right]^{2}\left(\frac{1}{\left(x_{i+1}-x\right)^{2 \mu}}+\frac{1}{\left(x-x_{i}\right)^{2 \mu}}\right) d x
\end{aligned}
$$

Recalling (7.1) with an appropriate scaling and applying the Bramble-Hilbert Theorem, we obtain

$$
\begin{aligned}
\int_{t_{i}}\left[\left(\psi-\psi_{h}\right)(x)\right]^{2}\left(\frac{1}{\left(x_{i+1}-x\right)^{2 \mu}}+\frac{1}{\left(x-x_{i}\right)^{2 \mu}}\right) d x & \leq C\left|\psi-\psi_{h}\right|_{H^{\mu}\left(t_{i}\right)}^{2} \\
& \leq C h^{2(\nu-\mu)}|\psi|_{H^{\nu}\left(t_{i}\right)}^{2}
\end{aligned}
$$


In view of this bound we deduce that

$$
\begin{aligned}
\sum_{i=0}^{i^{*}-1} \sum_{j \neq i} \int_{t_{i}} \int_{t_{j}} \frac{\left[\left(\psi-\psi_{h}\right)(x)-\left(\psi-\psi_{h}\right)(y)\right]^{2}}{|x-y|^{1+2 \mu}} d x d y & \leq C h^{2(\nu-\mu)} \sum_{i=0}^{i^{*}-1}|\psi|_{H^{\nu}\left(t_{i}\right)}^{2} \\
& \leq C h^{2(\nu-\mu)}|\psi|_{H^{\nu}(\Gamma)}^{2},
\end{aligned}
$$

which completes the proof.

Lemma 7.2. Let $\mu \in\left[0, \frac{1}{2}\left[\right.\right.$ and $\nu \in[\mu, 1]$. Then, $\forall \psi \in H^{\mu}(\Gamma)^{\prime}$,

$$
\left\|\psi-\pi_{h} \psi\right\|_{H^{\nu}(\Gamma)^{\prime}} \leq C h^{\nu-\mu}\|\psi\|_{H^{\mu}(\Gamma)^{\prime}} .
$$

Proof. Resorting to the duality of Aubin-Nitsche, we write

$$
\left\|\psi-\pi_{h} \psi\right\|_{H^{\nu}(\Gamma)^{\prime}}=\sup _{\chi \in H^{\nu}(\Gamma)} \frac{\left\langle\psi-\pi_{h} \psi, \chi\right\rangle_{\nu, \Gamma}}{\|\chi\|_{H^{\nu}(\Gamma)}}=\sup _{\chi \in H^{\nu}(\Gamma)} \frac{\left\langle\psi, \chi-\pi_{h} \chi\right\rangle_{\nu, \Gamma}}{\|\chi\|_{H^{\nu}(\Gamma)}} .
$$

Then by Lemma 7.1

$$
\left\langle\psi, \chi-\pi_{h} \chi\right\rangle_{\nu, \Gamma} \leq\|\psi\|_{H^{\mu}(\Gamma)^{\prime}}\left\|\chi-\pi_{h} \chi\right\|_{H^{\mu}(\Gamma)} \leq C h^{\nu-\mu}\|\psi\|_{H^{\mu}(\Gamma)^{\prime}}\|\chi\|_{H^{\nu}(\Gamma)} .
$$

Hence the proof.

\section{Appendix B}

Our aim here is to prove a sharp estimate used in the proof of Lemmas 4.11 and 5.4. Let $t$ be a finite segment of $\mathbb{R}$ and $h$ its length. Then

Lemma 8.1. For any $\left.\alpha \in] \frac{1}{2}, 1\right]$, there exists a constant $C>0$ independent of $h$ so that, $\forall \psi \in H^{\alpha}(t), \forall x_{0} \in t$,

$$
\left\|\psi-\psi\left(x_{0}\right)\right\|_{L^{2}(t)} \leq C h^{\alpha}|\psi|_{H^{\alpha}(t)} .
$$

Proof. Notice that this result is interesting in that the constant $C$ is uniform for arbitrary $x_{0}$. Let us first consider the reference segment $\hat{t}=(0,1)$. Then the Sobolev space $H^{\alpha}(\hat{t})$ is embedded in the space $\mathcal{C}(\hat{t})$ with a continuous embedding (see [1]) and therefore $\hat{\psi}\left(\hat{x}_{0}\right)$ makes sense. Then, we have in particular, $\forall \psi \in H^{\alpha}(\hat{t})$,

$$
\sup _{\hat{x}, \hat{y} \in \hat{t}}|\hat{\psi}(\hat{x})-\hat{\psi}(\hat{y})| \leq \hat{c}\|\hat{\psi}\|_{H^{\alpha}(\hat{t})} .
$$

or, again by the Bramble-Hilbert Theorem,

$$
\sup _{\hat{x}, \hat{y} \in \hat{t}}|\hat{\psi}(\hat{x})-\hat{\psi}(\hat{y})| \leq \hat{c} \inf _{d \in \mathbb{R}}\|\hat{\psi}-d\|_{H^{\alpha}(\hat{t})} \leq \hat{c}|\hat{\psi}|_{H^{\alpha}(\hat{t})} .
$$

Then, we derive that

$$
\left\|\hat{\psi}-\hat{\psi}\left(\hat{x}_{0}\right)\right\|_{L^{2}(\hat{t})} \leq\left\|\hat{\psi}-\hat{\psi}\left(\hat{x}_{0}\right)\right\|_{L^{\infty}(\hat{t})} \leq \sup _{\hat{x}, \hat{y} \in \hat{t}}|\hat{\psi}(\hat{x})-\hat{\psi}(\hat{y})| \leq \hat{c}|\hat{\psi}|_{H^{\alpha}(\hat{t})} .
$$

Hence the result for $\hat{t}$. A standard scaling argument lets us recover the result of the lemma with $C=\hat{c}$. 
Remark 8.2. This lemma plays a fundamental role in the proof of the optimality of the consistency error in Lemma 4.11 and of the approximation error in Lemma 5.4. When $\alpha \leq \frac{1}{2}$, the estimate no longer holds, and the incidence on the analysis of these errors when $u \in H^{\nu}(\Omega)$ with $0<\nu \leq 2$ is dramatic, and the techniques developed in this paper fail to recover the optimality, at least without an additional assumption (see Lemmas 4.12 4.13, 5.5, and 5.6).

\section{REFERENCES}

1. R. A. Adams. Sobolev Spaces, Academic Press, 1975. MR 56:9247

2. M. Benassi and R. E., White. Parallel Numerical Solution of Variational Inequalities, SIAM J. Numer. Anal., 31: 813-830, 1994. MR 95b:65165

3. F. Ben Belgacem. Numerical Simulation of some Variational Inequalities Arisen from Unilateral Contact Problems by the Finite Element Method, SIAM J. Numer. Anal., 37: 1198-1216, 2000. CMP 2000:12

4. F. Ben Belgacem. Mixed Finite Element Methods for Signorini's Problem, submitted.

5. F. Ben Belgacem ans S. C. Brenner. Some Nonstandard Finite Element Estimates with Applications to 3D Poisson and Signorini Problems, Electronic Transactions in Numerical Analysis, 12:134-148, 2001.

6. F. Ben Belgacem, P. Hild and P. Laborde. Extension of the Mortar Finite Element to a Variational Inequality Modeling Unilateral Contact, Math. Models and Methods in Appl. Sci., 9: 287-303, 1999. MR 99m:73066

7. C. Bernardi, Y. Maday et A. T. Patera. A New Non Conforming Approach to Domain Decomposition: The Mortar Element Method. Collège de France Seminar, (H. Brezis and J.-L. Lions, eds.), Pitman Res. Notes Math., vol. 299, Longman Sci. Tech., Harlow, 1994, pp. 13-51. MR 95a:65201

8. F. Brezzi, W. W. Hager and P. A. Raviart. Error estimates for the finite element solution of variational inequalities, Numer. Math., 28: 431-443, 1977. MR 56:7254

9. P.-G. Ciarlet. The Finite Element Method for Elliptic Problems, North Holland, 1978. MR 58:25001

10. P. Coorevits, P. Hild, K. Lhalouani and T. Sassi. Mixed Finite Element Method for Unilateral Problems: Convergence Analysis and Numerical Studies, Math. of Comp., posted on May 21, 2001, PII: S0025-5718(01)01318-7 (to appear in print).

11. G. Duvaut and J.-L. Lions. Les inéquations en mécanique et en physique, Dunod, 1972. MR 57:4778

12. R. S. Falk. Error Estimates for the Approximation of a Class of Variational Inequalities, Math. of Comp., 28: 963-971, 1974. MR 52:12323

13. R. Glowinski, J.-L. Lions and R. Trémolières. Analyse numériques des inéquations variationnelles, Tome 1, Dunod, 1976. MR 58:31697

14. P. Grisvard. Elliptic Problems in Nonsmooth Domains, Monographs and Studies in Mathematics, 24, Pitman, 1985. MR 86m:35044

15. J. Haslinger and I. Hlaváček. Contact between Elastic Bodies -2.Finite Element Analysis, Aplikace Matematiky, 26: 263-290, 1981. MR 83c:73052

16. J. Haslinger, I. Hlaváček and J. Nečas. Numerical Methods for Unilateral Problems in Solid Mechanics, in Handbook of Numerical Analysis, Vol. IV, Part 2, Eds. P.G. Ciarlet and J.-L. Lions, North Holland, 1996, pp. 313-485. MR 98b:65001

17. P. Hild. Problèmes de contact unilatéral et maillages incompatibles, Thèse de l'Université Paul Sabatier, Toulouse 3, 1998.

18. N. Kikuchi and J. T. Oden. Contact Problems in Elasticity: A Study of Variational Inequalities and Finite Element Methods, SIAM, 1988. MR 89j:73097

19. D. Kinderlehrer and G. Stamppachia. An Introduction to Variational Inequalities and their Applications, Academic Press, 1980. MR 81g:49013

20. K. Lhalouani and T. Sassi. Nonconforming Mixed Variational Formulation and Domain Decomposition for Unilateral Problems, East West J. Numer. Math., 7: 23-30, 1999. MR 2000b:65217

21. J.-L. Lions and E. Magenes. Problèmes aux limites non homogènes, Dunod, 1968. MR 40:512

22. M. Moussaoui and K. Khodja. Régularité des solutions d'un problème mêlé Dirichlet-Signorini dans un domaine polygonal plan, Commun. Part. Diff. Eq., 17: 805-826, 1992. MR 93h:35055 
23. G. Strang and G. J. Fix. An Analysis of the Finite Element Method, Prentice-Hall, 1973. MR 56:1747

24. Z.-H. Zhong. Finite Element Procedures for Contact-Impact Problems, Oxford University Press, 1993.

Méthodes Mathématiques pour l'Analyse des Systèmes, CNRS-UPRES-A-7035, Université de Metz, ISGMP, Batiment A, Ile du Saulcy, 57045 Metz, France

E-mail address: belhach@poncelet.sciences.univ-metz.fr

Mathématiques pour l'Industrie et la Physique, Unité Mixte de Recherche CNRSUPS-INSAT-UT1 (UMR 5640), Université Paul Sabatier, 118 route de Narbonne, 31062 Toulouse Cedex 04, France

E-mail address: belgacem@ip.ups-tlse.fr 